\title{
Theoretical investigation of amplified spontaneous emission with picosecond light pulses in dye solutions
}

\author{
A. PENZKOFER \\ Fachbereich Physik der Universität Regensburg, Germany \\ W. FALKENSTEIN \\ Physik Department der Technischen Universität München, Germany
}

Received 5 December 1977; revised 26 February 1978

\begin{abstract}
The amplified spontaneous emission in dye solutions excited with intense picosecond light pulses is studied theoretically. A multi-level model is applied to take into account the effects of the various dye parameters such as ground-state absorption, stimulated emission, excited-state absorption, reabsorption and relaxations from populated excited levels. The influence of the pump laser duration and intensity and of the dye concentration and sample length is investigated. Optimum situations are derived for the generation of intense picosecond light pulses at new frequencies by amplified spontaneous emission. On the other hand, conditions are found where amplification of spontaneous emission may be neglected. The analysis allows the determination of unknown dye parameters by comparing the calculations with the experiment.
\end{abstract}

\section{Introduction}

Amplification of spontaneous emission (superfluorescence) is readily observed in organic dye solutions when the sample is excited with intense light pulses [1-14]. The spontaneous fluorescence light is amplified by stimulated emission. When picosecond light pulses are used to excite the dye solutions, the fluorescence signal is shortened in time, increased in intensity, narrowed in spectral extension, and concentrated into a small divergence angle $[1-3,7,8,12,14]$. The effect of amplified spontaneous emission allows the generation of picosecond light pulses in new frequency regions. The conventional fluorescence studies are carried out with weak excitation light sources and the amplification effects are negligibly small; but the amplification of spontaneous emission alters the spectroscopic behaviour substantially when intense pump pulses are used $[15,16]$.

The purpose of this paper is to study, theoretically, the influence of various parameters on the fluorescence behaviour of dyes which are excited with intense picosecond light pulses. A model is used that allows the study of the influence of pump pulse absorption, stimulated emission, reabsorption and excited-state absorption as well as of relaxation phenomena in excited states. The effects of the geometrical arrangement, the pump pulse parameters and of the dye concentration are discussed. The analysis allows the determination of unknown dye parameters, such as relaxation rates, by comparing the calculations with the experiment. Optimum situations are found for the generation of picosecond light pulses at new frequencies. Conditions are derived where amplification of spontaneous emission is negligible and conventional fluorescence spectroscopy leads to correct results.

\section{Derivation of the set of equations}

The calculations are based on a multi-level model for the dye solution. The level scheme together with the various transitions is shown in Fig. 1. At the beginning, the molecules are in the singlet ground state 


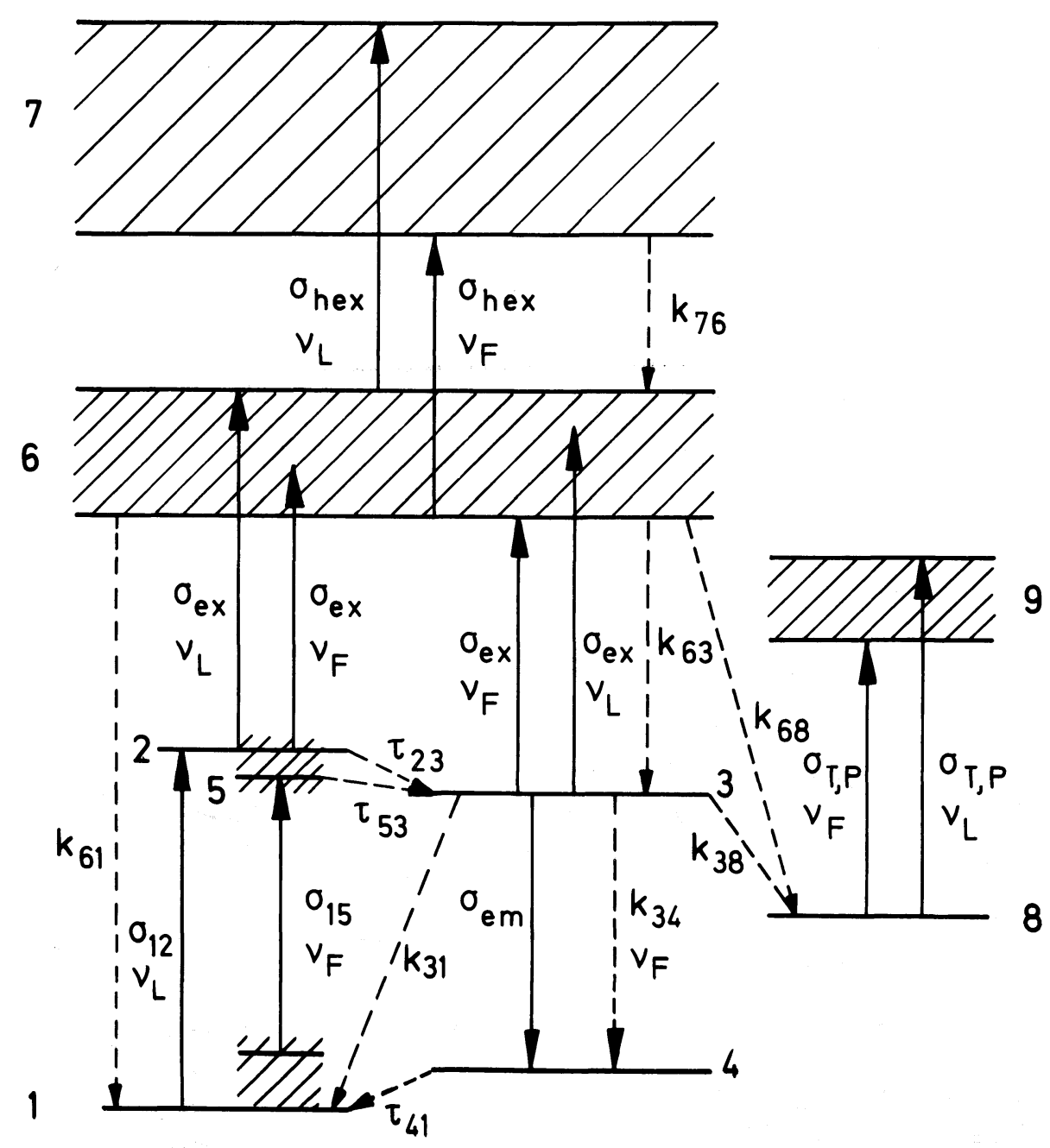

Figure 1 Multi-level system for the interaction of light with dye molecules. 1 , ground state; 2, Franck-Condon state in $S_{1}$-band; 3 , temporal equilibrium position in $S_{1}$-band; 4 , Franck-Condon state in $S_{0}$-band; 5 , region in $S_{1}$-band reached by reabsorption of fluorescence light; 6 and 7 , higher singlet states populated by excited-state absorption; 8 , triplet state or state of photodecomposed molecules; 9 , excited states in the triplet system or in the decomposed system. The solid arrows indicate absorption and stimulated emission processes, the dashed arrows illustrate the various relaxation channels.

(Level 1). An intense picosecond light pulse (frequency $\nu_{\mathrm{L}}$ ) excites part of the molecules to a vibrational Franck-Condon state in the $S_{1}$-singlet band (Level 2). Within the $S_{1}$-band the molecules relax (time constant $\tau_{23}$ ) to a temporal equilibrium position (3). From this position, spontaneous emission to the Franck-Condon state (4) in $\mathrm{S}_{0}$ occurs with a rate constant $k_{34}$ (equal to the Einstein transition probability $\boldsymbol{A}_{34}$ ). The spontaneous emission initiates the stimulated emission between Levels 3 and 4 . Part of the molecules in the $S_{1}$ state decay radiationlessly with a rate constant $k_{31}$ to the ground state. The inter-system crossing to the triplet system (Level 8) is taken into account by the rate constant $k_{38}$. Molecules in the $\mathrm{S}_{1}$-band (Levels 2 and 3 ) absorb pump light and fluorescence light by excited-state absorption. The dye molecules are thereby excited to higher lying singlet states (6). A constant absorption cross-section $\sigma_{\text {ex }}$ for the transitions from Levels 2 and 3 to Band 6 is assumed in most of our calculations. The molecules in Band 6 relax within the singlet-state system to Level 3 (rate $k_{63}$ ) and Level 1 (rate $k_{61}$ ) or they are transferred to triplet states or are decomposed (Level 8). The population of the 
triplet states (inter-system crossing) and the photodecomposition are taken into account by a single rate constant $k_{68}$. The absorption of the molecules in the triplet state and of the decomposed molecules is assumed to be $\sigma_{\mathrm{T}, \mathrm{P}}$. With intense picosecond pump pulses, even the absorption from high-lying singlet states (6) to higher states (7) has to be considered (absorption cross-section $\sigma_{\text {hex }}$ ). In most of our calculations we assume a constant absorption cross-section $\sigma_{\mathrm{ex}}$ for all molecules in an excited singlet state $\left(S_{n}, n \geqslant 1\right)$. Part of the fluorescence light is reabsorbed and thereby transfers thermally-excited molecules in the $S_{0}$-band to the $S_{1}$-band (5). (The fluorescence frequencies overlap with the long-wavelength shoulder of the $S_{0}-S_{1}$ absorption spectra of the dyes).

In our calculations we used parameters which apply to rhodamine $6 \mathrm{G}$ dissolved in ethanol, a system we investigated extensively [17]. The parameters were individually varied to study their influence on the effect of amplified spontaneous emission.

The geometrical model used in the calculations is adapted to our experimental arrangement [17]. A single picosecond pump pulse passes through a tilted dye cell (longitudinal excitation). The use of short excitation pulses $\left(\Delta t_{\mathrm{L}} c_{0} \ll l\right.$, where $l$ is the cell length, $c_{0}$ the velocity of light) allows us to reduce the discussion of amplified spontaneous emission to the forward propagation direction (travelling-wave generation of stimulated fluorescence light). As long as the stimulated emission is weak, the amplification in the backward direction does not affect the forward light generation. In strong forward stimulated emission the fluorescence emission is shortened to the picosecond range and the backward emission becomes small compared to the forward emission [1] (shorter interaction length in backward direction). The tilting of the dye cell eliminates the complicating influence of back-reflections of fluorescence light $[1,17]$.

The pump pulse initiates spontaneous emission in all directions. The spontaneously emitted photons travelling along the path of the exciting pulse are amplified by stimulated emission. We assume a constant gain factor within a divergence angle which is determined by the pump pulse diameter and the shorter of either the cell length or the absorption depth (length within which the pump pulse is reduced to $1 / \mathrm{e}$ of its initial power). A constant pump pulse intensity and a constant excited-state population within the spatial beam profile are assumed for simplicity.

The fluorescence light is emitted within a broad spectral range. The stimulated emission narrows the spectral extension [18-20]. In our calculations we do not study spectral narrowing. A constant emission cross-section for all frequencies is assumed and we discuss in a semi-quantitative manner only the effects of spectral narrowing by amplification of spontaneous emission.

The absorption cross-section of the dye molecules depends on their orientation relative to the pump light polarization [21]. When the angle between the transition moment of the molecule and the light polarization vector is $\theta$ then the absorption cross-section is given by [22]

$$
\tilde{\sigma}(\theta)=\sigma_{\|} \cos ^{2} \theta+\sigma_{\perp} \sin ^{2} \theta
$$

[the isotropic absorption cross-section is $\sigma=\int \tilde{\sigma}(\theta) \mathrm{d} \theta=\frac{1}{3}\left(\sigma_{\|}+2 \sigma_{\perp}\right)$ ]. The excitation of dye solutions with linearly polarized light pulses generates an anisotropic orientation of the excited molecules in the $\mathrm{S}_{1}$-band (Levels 2 and 3). The excited molecules emit light polarized in the plane formed by the transition moment and the propagation direction. The emission cross-section depends on the angle $\psi$ between the transition vector and the propagation direction in the form [23]

$$
\tilde{\sigma}_{\mathrm{em}}(\psi)=\tilde{\sigma}_{\mathrm{em}}(0) \sin ^{2} \psi .
$$

In our calculations we take into account only the anisotropic absorption of the pump pulse. But we neglect the anisotropic emission effects for the following two reasons:

(a) When weak excitation pulses are used, the fluorescence decay time is long compared to the reorientation time of excited molecules in low viscous solvents [24, 16, 22, 25]. For rhodamine 6G dissolved in ethanol the reorientation time constant is 270 ps while the spontaneous fluorescence decay time is about $4.4 \mathrm{~ns}$. An average emission cross-section $\sigma_{\mathrm{em}}=\tilde{\sigma}_{\mathrm{em}}(\theta=0) / 3$ leads to reasonable results.

(b) When intense excitation pulses are applied, nearly all molecules in the ground state with their 
different orientations are excited and an isotropic distribution of molecules in the excited state is approached $[22,25]$.

In our calculations we use rate equations to describe the interaction of pump and fluorescence light with the dye molecules. The rate equation approach is applicable for pulse durations which are long compared to the dephasing time $T_{2}$ of the dye molecules. This condition is fulfilled in our experiments (pump pulse duration $\Delta t_{\mathrm{L}} \simeq 4 \mathrm{ps,} \mathrm{dephasing} \mathrm{time} T_{2} \simeq 0.5 \mathrm{ps}$ [26]).

With the assumptions and approximations described above we write down the rate equations for the absorption and emission processes of Fig. 1. The equations for the populations of the various levels and for the pump light and fluorescence light are as follows:

$$
\begin{aligned}
& \frac{\partial N_{1}(\theta)}{\partial t}=-\frac{I_{\mathrm{L}}}{h \nu_{\mathrm{L}}}\left(\sigma_{\| 12} \cos ^{2} \theta+\sigma_{\perp 12} \sin ^{2} \theta\right)\left[N_{1}(\theta)-N_{2}(\theta)\right]-\sigma_{15} \frac{I_{\mathrm{F}}}{h \nu_{\mathrm{F}}}\left[N_{1}(\theta)-N_{5}\right]+\frac{N_{4}}{\tau_{41}} \\
& +k_{61} N_{6}+k_{31}\left[N_{2}(\theta)+N_{3}+N_{5}\right] \\
& \frac{\partial N_{2}(\theta)}{\partial t}=\frac{I_{\mathrm{L}}}{h \nu_{\mathrm{L}}}\left\{\left(\sigma_{\| 12} \cos ^{2} \theta+\sigma_{\perp 12} \sin ^{2} \theta\right)\left[N_{1}(\theta)-N_{2}(\theta)\right]-\left(\sigma_{\| \operatorname{ex}} \cos ^{2} \theta+\sigma_{\perp \text { ex }} \sin ^{2} \theta\right)\left[N_{2}(\theta)\right.\right. \\
& \left.\left.-N_{6} \frac{N_{2}}{N_{2}+N_{3}} \frac{I_{\mathrm{L}}}{I_{\mathrm{L}}+I_{\mathrm{F}}}\right]\right\}-\frac{I_{\mathrm{F}}}{h \nu_{\mathrm{F}}}\left(\sigma_{\| \operatorname{ex}} \cos ^{2} \theta+\sigma_{\perp \text { ex }} \sin ^{2} \theta\right)\left[N_{2}(\theta)-N_{6} \frac{N_{2}}{N_{2}+N_{3}} \frac{I_{\mathrm{F}}}{I_{\mathrm{L}}+I_{\mathrm{F}}}\right] \\
& -\frac{N_{2}(\theta)}{\tau_{23}} \\
& N_{1}=\int_{0}^{\pi / 2} N_{1}(\theta) \sin \theta \mathrm{d} \theta \\
& N_{2}=\int_{0}^{\pi / 2} N_{2}(\theta) \sin \theta \mathrm{d} \theta \\
& \frac{\partial N_{3}}{\partial t}=-\sigma_{\mathrm{em}} \frac{I_{\mathrm{F}}}{h \nu_{\mathrm{F}}}\left(N_{3}-N_{4}\right)-\sigma_{\mathrm{ex}}\left[\frac{I_{\mathrm{L}}}{h \nu_{\mathrm{L}}}\left(N_{3}-N_{6} \frac{N_{3}}{N_{2}+N_{3}} \frac{I_{\mathrm{L}}}{I_{\mathrm{L}}+I_{\mathrm{F}}}\right)\right. \\
& \left.+\frac{I_{\mathrm{F}}}{h \nu_{\mathrm{F}}}\left(N_{3}-N_{6} \frac{N_{3}}{N_{2}+N_{3}} \frac{I_{\mathrm{F}}}{I_{\mathrm{L}}+I_{\mathrm{F}}}\right)\right]+\frac{N_{2}}{\tau_{23}}+\frac{N_{5}}{\tau_{53}}+k_{63} N_{6} \\
& -\left(k_{31}+k_{34}+k_{38}\right) N_{3} \\
& \frac{\partial N_{4}}{\partial t}=\sigma_{\mathrm{em}} \frac{I_{\mathrm{F}}}{h \nu_{\mathrm{F}}}\left(N_{3}-N_{4}\right)+k_{34} N_{3}-\frac{N_{4}}{\tau_{41}} \\
& \frac{\partial N_{5}}{\partial t}=\sigma_{15} \frac{I_{\mathrm{F}}}{h \nu_{\mathrm{F}}}\left(N_{1}-N_{5}\right)-\left(\frac{1}{\tau_{53}}+k_{31}\right) N_{5} \\
& \frac{\partial N_{6}}{\partial t}=\sigma_{\mathrm{ex}}\left[\frac{I_{\mathrm{L}}}{h \nu_{\mathrm{L}}}\left(N_{2}+N_{3}-N_{6} \frac{I_{\mathrm{L}}}{I_{\mathrm{L}}+I_{\mathrm{F}}}\right)+\frac{I_{\mathrm{F}}}{h \nu_{\mathrm{F}}}\left(N_{2}+N_{3}-N_{6} \frac{I_{\mathrm{F}}}{I_{\mathrm{L}}+I_{\mathrm{F}}}\right)\right]-\left(k_{61}+k_{63}+k_{68}\right) N_{6} \\
& \frac{\partial N_{8}}{\partial t}=k_{38} N_{3}+k_{68} N_{6} \\
& \frac{\partial I_{\mathbf{L}}}{\partial x}+\frac{\eta}{c_{0}} \frac{\partial I_{\mathbf{L}}}{\partial t}=-I_{\mathrm{L}}\left(\int _ { 0 } ^ { \pi / 2 } \left\{\left(\sigma_{\| 12} \cos ^{2} \theta+\sigma_{\perp 12} \sin ^{2} \theta\right)\left[N_{1}(\theta)-N_{2}(\theta)\right]\right.\right. \\
& \left.\left.+\left(\sigma_{\| \text {ex }} \cos ^{2} \theta+\sigma_{\perp \text { ex }} \sin ^{2} \theta\right)\left[N_{2}(\theta)+N_{3}\right]\right\} \sin \theta \mathrm{d} \theta+\sigma_{\mathrm{T} . \mathrm{P}} N_{\mathbf{8}}\right)
\end{aligned}
$$




$$
\frac{\partial I_{\mathrm{F}}}{\partial x}+\frac{\eta}{c_{0}} \frac{\partial I_{\mathrm{F}}}{\partial t}=\frac{h \nu_{\mathrm{F}} k_{34} N_{3} \Delta \Omega}{4 \pi}+I_{\mathrm{F}}\left[\sigma_{\mathrm{em}}\left(N_{3}-N_{4}\right)-\sigma_{\mathrm{ex}}\left(N_{2}+N_{3}\right)-\sigma_{15}\left(N_{1}-N_{5}\right)-\sigma_{\mathrm{T}, \mathrm{P}} N_{8}\right] .
$$

In Equation 1 the first term describes the absorption of the pump pulse. The second term represents the reabsorption of fluorescence light. The last three terms take into account the relaxation (radiationless and radiating) from excited levels to the ground state.

The first term in Equation 2 gives the population of Level 2 by pump pulse absorption. The excitedstate absorption of laser and fluorescence light is described by the second and third terms. The laser and the fluorescence light excite the molecules to different levels in Band 6. The correction factors to $N_{6}$ take care of this fact in Equation 2 and in the following equations. The last term of Equation 2 is responsible for the fast relaxation to the temporal equilibrium state ( 3 in Fig. 1).

Equations 3 and 4 calculate the average population number density in the ground state and the excited Franck-Condon state.

Equation 5 is determined by the stimulated emission (first term), the excited-state absorption (terms in square brackets), the decay to Level 3 within the $S_{1}$-band (next two terms) and from the higher-lying Levels 6 (fifth term). The last term represents the depopulation of Levels 3 by internal conversion $\left(k_{31}\right)$, by spontaneous emission $\left(k_{34}\right)$ and by inter-system crossing $\left(k_{38}\right)$.

Equation 6 shows that stimulated emission and spontaneous emission populate Level 4 while the radiationless decay with the time constant $\tau_{41}$ empties it.

The reabsorption of fluorescence light populates low-lying states (5) in the $S_{1}$-band as described by Equation 7. (The short-wavelength shoulder of the emission spectrum overlaps somewhat with the long-wavelength tail of the absorption spectrum). The molecules in Level 5 relax to the temporal equilibrium position (3) $\left(\tau_{53}=\tau_{23}\right.$ is assumed in our calculations) or return to the ground state (1) by internal conversion.

The population of the higher-lying singlet Band 6 (Equation 8) is caused by excited-state absorption of laser and fluorescence light starting from Levels 2 and 3 (terms in square brackets). Excitation to even higher-lying states (7) may be included using a similar term (see later). The last term is responsible for the relaxation from Level 6 to lower levels.

Equation 9 determines the population of triplet states and of decomposition states.

The absorption of the pump pulse is described by Equation 10. The first term under the integral is responsible for ground-state absorption. The second term takes care of the excited-state absorption. The absorption between the $\mathrm{S}_{1}$-band (Levels 2 and 3) and Band 6 should be proportional to $N_{2}(\theta)+N_{3}-N_{6}$. Generally, we consider a constant absorption cross-section $\sigma_{\mathrm{ex}}$ for all molecules in excited singlet states (Levels 2, 3, 6 and 7). For this reason the term $N_{6}$ is dropped in the second term. The effects of excitedstate absorption between Bands 6 and 7 with $\sigma_{\mathrm{ex}} \neq \sigma_{\text {hex }}$ will be discussed later. The last term in Equation 10 is due to the triplet-state absorption and to the absorption of decomposed molecules.

The build-up of fluorescence light in the forward direction is given by Equation 11. The spontaneous emission within a solid angle $\Delta \Omega$ around the forward direction (first term in Equation 11) is amplified by stimulated emission (first term in the square brackets). Excited-state absorption processes ( $\sigma_{\mathrm{ex}}$ and $\left.\sigma_{\mathrm{T}, \mathrm{P}}\right)$ reduce the fluorescence light generation.

Equations 1-11 are solved by numerical methods. The system is simplified by the transformation $z=x$ and $t^{\prime}=t-\left(\eta / c_{0}\right) x$ which changes the derivatives to $\partial / \partial t=\partial / \partial t^{\prime}$ and $\partial / \partial x+\left(\eta / c_{0}\right) \partial / \partial t=\partial / \partial z$. The initial condition for the populations of the levels are

and

$$
N_{1}\left(t^{\prime}=-\infty, z, \theta\right)=N
$$

$$
N_{2}\left(t^{\prime}=-\infty, z, \theta\right)=\ldots=N_{8}\left(t^{\prime}=-\infty, z\right)=0 .
$$

The incident pump pulse is given by

$$
I_{\mathrm{L}}\left(t^{\prime}, z=0\right)=I_{0 \mathrm{~L}} \exp \left(-t^{\prime 2} / t_{0}^{2}\right)
$$

At the beginning, when no fluorescence light is present, $I_{\mathrm{F}}\left(t^{\prime}, z=0\right)=0$. 


\section{Discussion}

The laser parameters, geometrical factors and dye characteristics are chosen to correspond to our experimental studies [17]. The influence of the various parameters on the amplified spontaneous emission is studied by varying one parameter and keeping all other parameters constant. The applied picosecond light pulses have a frequency of $\widetilde{\nu}_{\mathrm{L}}=18910 \mathrm{~cm}^{-1}$ (second harmonic pulses of a modelocked Nd-silicate glass laser). The standard pulse duration is $\Delta t_{\mathrm{L}}=4 \mathrm{ps}$. The tilted dye cell has an active length of $1.13 \mathrm{~cm}$. The spontaneous emission is assumed to be homogeneously amplified within a standard solid angle of $\Delta \Omega=2.5 \times 10^{-3} \mathrm{sr}$. The chosen dye parameters apply to rhodamine $6 \mathrm{G}$ dissolved in ethanol and are listed in Table I. It should be mentioned that in the experiments $\Delta \Omega$ depends on the penetration depth of the laser beam while $\sigma_{\mathrm{em}}$ and $\sigma_{15}$ depend on the spectral shift of the fluorescence emission. These effects, and consequently the values of $\Delta \Omega, \sigma_{\mathrm{em}}$ and $\sigma_{15}$, vary with dye concentration and laser intensity.

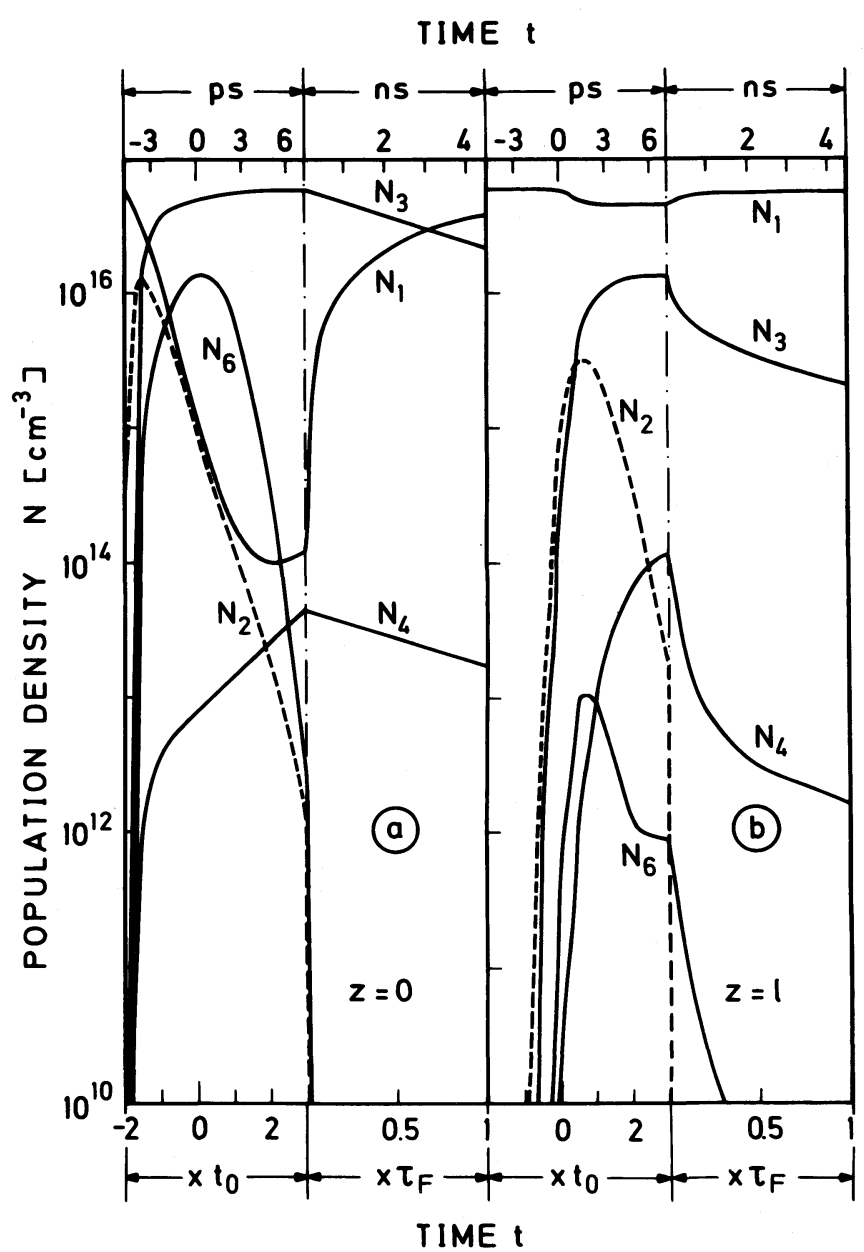

Figure 2 Time-dependent variation of the population of the various states of the multi-level system. (a) At the beginning of the sample $z=0$. (b) At the end of the sample $z=1$. Input pump pulse intensity $I_{0}=2 \times 10^{10} \mathrm{~W} \mathrm{~cm}^{-2}, t_{0}=$ $\Delta t_{L} / 2 \sqrt{ } \ln 2$. The dye parameters listed in Table I apply to all figures, except otherwise mentioned. For all figures the sample length is $l=1.13 \mathrm{~cm}$ and the solid angle is $\Delta \Omega=2.5 \times 10^{-3} \mathrm{sr}$ (except variation in Figs. $5 \mathrm{~b}$ and 6 ). 
TAB LE I Chosen dye parameters (Rhodamine 6G in ethanol)

\begin{tabular}{|c|c|}
\hline $\begin{array}{l}\text { Absorption cross-sections } \\
\left(\mathrm{cm}^{2}\right)\end{array}$ & Decay rate \\
\hline $\begin{array}{ll}\tilde{\sigma}_{12}=3 \sigma_{12} & =1.25 \times 10^{-15} \mathrm{a} \\
\sigma_{\|_{12}} & =1.17 \times 10^{-15} \mathrm{~b} \\
\sigma_{\perp_{12}} & =4.2 \times 10^{-17} \mathrm{~b} \\
\tilde{\sigma}_{\mathrm{ex}}=3 \sigma_{\mathrm{ex}} & =1.8 \times 10^{-16} \mathrm{a} \\
\sigma_{\| \mathrm{ex}} & =1.68 \times 10^{-16} \mathrm{~b} \\
\sigma_{\perp \mathrm{ex}} & =6.1 \times 10^{-18} \mathrm{~b} \\
\sigma_{\text {hex }}=\sigma_{\mathrm{ex}} \mathrm{c} & \\
\sigma_{\text {em }} & =2.5 \times 10^{-16} \mathrm{~d} \\
\sigma_{15} & \mathrm{e} \\
\sigma_{\mathrm{T}, \mathrm{P}} & =0\end{array}$ & $\begin{array}{l}\tau_{23}=9 \times 10^{-13} \mathrm{~s}^{2, \mathrm{~b}} \\
\tau_{41}=4 \times 10^{-12} \mathrm{~s}^{\mathrm{g}} \\
k_{31}=2.5 \times 10^{7} \mathrm{~s}^{-1} \mathrm{~h} \\
k_{34}=2 \times 10^{8} \mathrm{~s}^{-1} \mathrm{~h} \\
k_{38}=0^{i} \\
k_{61}=0^{\mathrm{e}} \\
k_{63}=10^{13} \mathrm{~s}^{-1} \mathrm{j} \\
k_{68}=0^{\mathrm{e}} \\
\text { emission frequency } 17700 \mathrm{~cm}^{-1} \\
\text { concentration } 10^{-4} \mathrm{M}\end{array}$ \\
\hline $\begin{array}{l}\text { b see [ } 22] \\
\text { constant absorption cross-sectio } \\
\text { assumed. } \\
\text { dhe stimulated emission cross-se } \\
g(\widetilde{v}) q_{\mathrm{F}} k_{34} /\left(8 \pi \eta^{2} \widetilde{\nu}^{2}\right) \cdot g(\widetilde{\nu}) \text { desc } \\
\text { emission } \int g(\widetilde{v}) \mathrm{d} \widetilde{\nu}=1 ; q_{\mathrm{F}}=0 \\
1.36 \text { refractive index. A maximu } \\
\text { mated from the parameters. } \\
\text { preset; values are determined in } \\
\text { f for comparison see [28] } \\
\text { g see [29] } \\
\text { h see [30] } \\
\text { i A value of } k_{38}=4.2 \times 10^{5} \mathrm{~s}^{-1} \text { is } \\
\text { j see [31] and [17] }\end{array}$ & $\begin{array}{l}\text { ll excited molecules in the singlet system is } \\
\text { is calculated by the relation [27] } \sigma_{\mathrm{em}}(\widetilde{\mathcal{V}})= \\
\text { the spectral distribution of the fluorescence } \\
\text { the fluorescence quantum efficiency; } \eta= \\
\text { lue } \sigma_{\mathrm{em}}\left(\widetilde{\nu}_{\max }\right) \simeq 2.7 \times 10^{-16} \mathrm{~cm}^{2} \text { is esti- }\end{array}$ \\
\hline
\end{tabular}

\subsection{Temporal development}

The time dependence of the population of the various states of the dye system $\left(10^{-4} \mathrm{M}\right)$ of the sample at the beginning and at the end is shown in Figs. $2 a$ and $b$ for an incident pump pulse of peak intensity $I_{0 \mathrm{~L}}=2 \times 10^{10} \mathrm{~W} \mathrm{~cm}^{-2}$. The input pulse and the transmitted pulse together with the build-up of the fluorescence light are shown in Figs. $3 \mathrm{a}$ and $\mathrm{b}$ for input peak intensities of $I_{0 \mathrm{~L}}=2 \times 10^{10} \mathrm{~W} \mathrm{~cm}^{-2}$ and $10^{8} \mathrm{~W} \mathrm{~cm}^{-2}$, respectively. In the calculations the standard parameters cited above were used.

In Fig. 2a the temporal development of the populations of the various states at the beginning of the sample is depicted for intense input pulses. The ground-state population, $N_{1}$, is drastically decreased by pump pulse absorption. After the passage of the exciting pulse the ground state is repopulated by radiative and radiationless relaxation. The population, $N_{2}$, of the Franck-Condon level in the $\mathrm{S}_{1}$ state (broken line) rises steeply to a maximum value before the pump pulse reaches its maximum. The fast relaxation within the $\mathrm{S}_{1}$-band $\left(\tau_{23}\right)$, the increased excited-state absorption [proportional to $N_{2}(t) \times I_{\mathrm{L}}(t)$ ] and the depletion of the ground state, $N_{1}(t)$, terminate the $N_{2}$ values and cause their fast reduction. The temporal equilibrium state (3) in the $S_{1}$-band is populated by relaxation of the molecules in the excited Level 2. The excited-state absorption from Level 3 to Level 6 does not influence the $N_{3}$ value severely, since the molecules in Level 6 relax very fast back to Level 3. During and after the excitation, the molecules in the $S_{1}$-band decay to the Franck-Condon Level 4 in the $S_{0}$-band by spontaneous emission $\left(k_{34}\right)$. The molecules in Level 4 return to the ground state $(1)$ by radiationless processes. The population of Level 6 by excited-state absorption becomes rather large at the peak of the pump pulse. The absorption of laser light from Level 6 to higher states is taken into account by assuming a constant absorption cross-section, $\sigma_{\text {ex }}$, for all molecules in the $S_{1}$-band and in higher levels.

The time dependence of the population of the sample at the end (Fig. 2b) is qualitatively similar to 


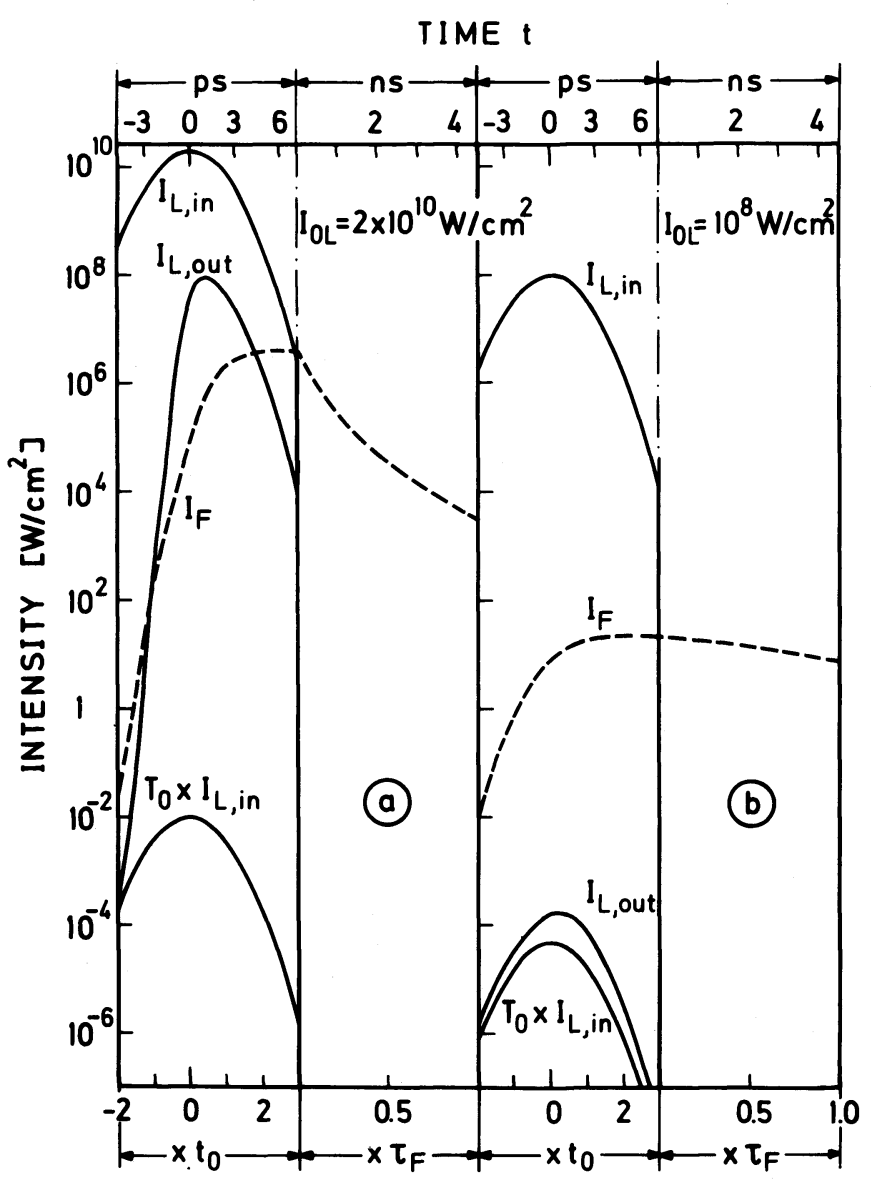

TIME $t$

Figure 3 Time dependence of the input pump pulse $/_{L}$,in, the transmitted pump pulse $/_{L}$,out, and the generated fluorescence pulse $I_{F}$. The expected output pump-pulse intensity $T_{0} \times I_{L}$,in in the case of no bleaching is included.

the beginning. The pump pulse of $I_{0 \mathrm{~L}}=2 \times 10^{10} \mathrm{~W} \mathrm{~cm}^{-2}$ is absorbed to a peak value of approximately $10^{8} \mathrm{~W} \mathrm{~cm}^{-2}$ (see Fig. 3a). The smaller pump intensity reduces the depletion of the ground state $\left(N_{1}\right)$. The $S_{1}$-state populations (Level 2 and 3 ) are correspondingly weaker. The effects of excited-state absorption become negligibly small and the population of Level 6 goes down drastically since $I_{\mathrm{L}}(t)$ and $N_{2}(t)+N_{3}(t)$ are reduced. The spontaneous fluorescence signal is amplified along the sample length by stimulated emission and reaches a value of several $\mathrm{MW} \mathrm{cm}^{-2}$. The maximum fluorescence is achieved towards the end of the pulse where the population of the emitting Level 3 is maximal. The stimulated emission enhances the depopulation of Level 3 as is clearly seen in Fig. 2b for times $t$ slightly longer than the pump pulse. The decrease of $N_{3}$ reduces the stimulated emission and an exponential relaxation determined by the spontaneous emission is approached.

In Figs. $3 \mathrm{a}$ and $\mathrm{b}$ the time dependence of the transmitted pump pulse $I_{\mathrm{L}, \text { out }}$ and of the generated fluorescence pulse $I_{\mathrm{F}}$ (at the end of the cell) are depicted for input peak intensities of $I_{0 \mathrm{~L}}=$ $2 \times 10^{10} \mathrm{~W} \mathrm{~cm}^{-2}$ and $10^{8} \mathrm{~W} \mathrm{~cm}^{-2}$, respectively. The input pulse shapes $I_{\mathrm{L} \text {, in }}$ and the output pulse shapes $T_{0} \times I_{\mathrm{L} \text {, in }}$ which were expected for small-signal absorption are included. For $I_{0 \mathrm{~L}}=2 \times 10^{10} \mathrm{~W} \mathrm{~cm}^{-2}$ the output pulse reaches a peak value which is a factor of $10^{10}$ larger than expected for small-signal absorption. This number indicates a strong bleaching of the dye. At an input peak intensity of $I_{0 \mathrm{~L}}=$ 
$10^{8} \mathrm{~W} \mathrm{~cm}^{-2}$, the pulse suffers strong absorption and is close to the small-signal case. The behaviour of the fluorescence light changes with the intensity of the exciting pulse. The fluorescence signal increases strongly with pump intensity due to the amplification of spontaneous emission. At $I_{0 \mathrm{~L}}=10^{8} \mathrm{~W} \mathrm{~cm}^{-2}$ the fluorescence light decays exponentially (spontaneous emission) after the excitation pulse has passed. At $I_{0 \mathrm{~L}}=2 \times 10^{10} \mathrm{~W} \mathrm{~cm}^{-2}$ the emitted pulse decays very fast in a non-exponential manner, due to the fast depletion of the $N_{3}$-population by stimulated emission. In Fig. $3 \mathrm{~b}$ the fluorescence pulse has a duration $(\mathrm{FWHM})$ of $\Delta t_{\mathrm{F}} \simeq 3 \mathrm{~ns}$ while in Fig. $3 \mathrm{a}$ the fluorescence is reduced to about $\Delta t_{\mathrm{F}} \simeq 170 \mathrm{ps}$.

\subsection{Effects of various parameters}

The efficiency of amplified spontaneous emission is influenced by pump pulse parameters (intensity, duration, diameter) by geometrical factors (sample length) and by dye characteristics (concentration, absorption cross-section, emission cross-section, rate constants). The influence of all these parameters on the amplification is studied by varying one parameter and keeping all the other parameters constant (see values in Table I). The fluorescence energy, $E_{\mathrm{F}}$, emitted in the forward direction, the fluorescence pulse duration $\Delta t_{\mathrm{F}}(\mathrm{FWHM})$ and the energy transmission $T_{\mathrm{E}}=E_{\mathrm{L} \text {, out }} / E_{\mathrm{L} \text {, in }}$ of the pump pulse are studied. In the following figures the fluorescence energy, $E_{\mathrm{F}}$, emitted within the solid angle $\Delta \Omega$ is normalized to the absorbed pump pulse energy $E_{\mathrm{abs}}=\left(1-T_{\mathrm{E}}\right) E_{\mathrm{L}, \text { in }}$, i.e. $\vec{E}_{\mathrm{F}}=E_{\mathrm{F}} / E_{\mathrm{abs}}$ is depicted.

\subsubsection{Effects of pump pulse parameters}

3.2.1.1. The influence of the pump pulse duration $\Delta t_{\mathrm{L}}(\mathrm{FWHM})$ on $\bar{E}_{\mathrm{F}}, T_{\mathrm{E}}$ and $\Delta t_{\mathrm{F}}$ are shown in Fig. $4 \mathrm{a}, \mathrm{b}$ and Fig. 5a, respectively. For longer laser pulses more dye molecules are excited at a fixed intensity. The amplified fluorescence is increased (Fig. 4a) and the fluorescence duration is shortened (Fig. 5a). Longer pump pulses bleach the dye harder and a higher energy transmission $T_{\mathbf{E}}$ results (Fig. $4 \mathrm{~b}$ ). The obtainable maximum fluorescence energy $E_{F}$ is determined by the available dye molecules (concentration) and is independent of pump pulse durations. Longer excitation pulses reach the maximum $\vec{E}_{\mathrm{F}}$ value at lower intensities (more photons at a fixed intensity). The reduction of the normalized fluorescence output $\bar{E}_{\mathrm{F}}=E_{\mathrm{F}} / E_{\text {abs }}$ at very high intensities is due to excited-state absorption and will be

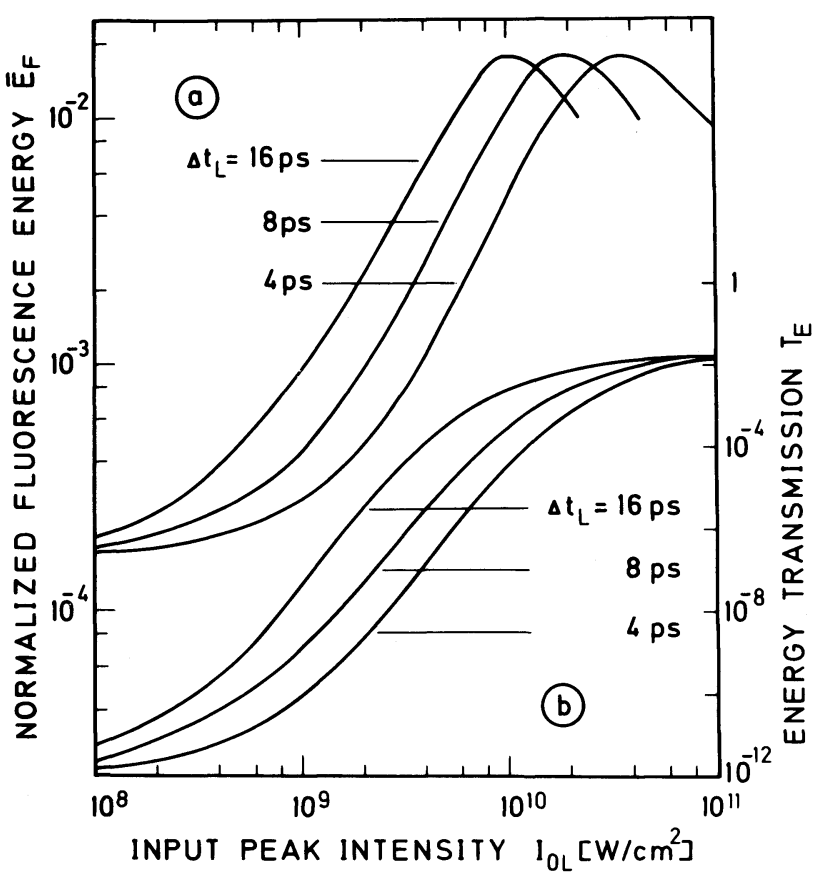

Figure 4 Effects of pump-pulse duration on amplification of fluorescence output (a) and on energy transmission of the exciting pulse (b). 


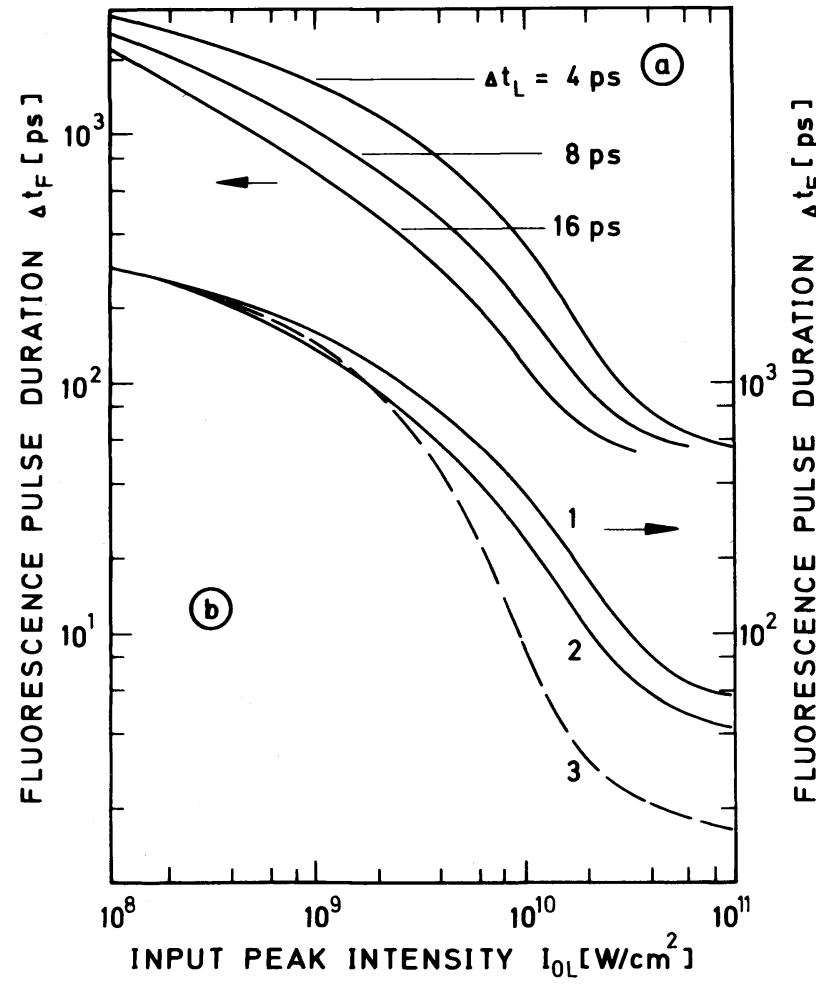

Figure 5 Pulse shortening of the amplified spontaneous emission. (a) Influence of the pump pulse duration. (b) Effects of reabsorption (Curves 1 and 2) and of solid angle of emission (Curves 1 and 3 ). Curve 1, $\sigma_{15}=0$ and $\Delta \Omega=2.5 \times 10^{-3} \mathrm{sr}$. Curve 2, $\sigma_{15}=$ $8 \times 10^{-17} \mathrm{~cm}^{2}$ and $\Delta \boldsymbol{Q}=2.5 \times 10^{-3} \mathrm{sr}$. Curve $3, \sigma_{15}=0$ and $\Delta \Omega=10^{-1} \mathrm{sr}$.

discussed later. The minimum fluorescence pulse duration at high pump intensities is approximately independent of the pump pulse duration as long as the longest $\Delta t_{\mathbf{L}}$ value is shorter than the minimum $\Delta t_{\mathrm{F}}$ value.

3.2.1.2. The effect of the solid angle $\Delta \Omega$ on the normalized fluorescence output $\bar{E}_{\mathrm{F}}$ is shown in Fig. 6 . At low pump intensities amplification of the spontaneously-emitted light is negligibly small and the fluorescence light is emitted nearly isotropically in all directions. The fluorescence energy within $\Delta \Omega$ is approximately given by $\bar{E}_{\mathrm{F}}=q_{\mathrm{F}} \Delta \Omega / 4 \pi$

where

$$
q_{\mathrm{F}}=k_{34} /\left(k_{34}+k_{31}+k_{38}\right)=k_{34} / k_{3}
$$

is the fluorescence quantum efficiency. As a result, the fluorescence yield is proportional to $\Delta \Omega$ at low pump intensities. At high pump intensities the total fluorescence output $\bar{E}_{\mathrm{F}}$ increases only slightly with $\Delta \Omega$ and the amplification $\bar{E}_{\mathrm{F}}\left(I_{\mathrm{L}}\right) / \bar{E}_{\mathrm{F}}(0)$ is reduced. The amplification of spontaneous emission within a large divergence angle depletes the population of the emitting Level 3 faster and causes a decrease of amplification together with a shortening of $\Delta t_{\mathrm{F}}$ (see dashed curve in Fig. $5 \mathrm{~b}$ ). The energy transmission $T_{\mathrm{E}}$ is found to be independent of $\Delta \Omega$.

In the experiments the solid angle $\Delta \Omega$, within which amplified spontaneous emission occurs, depends on pump pulse and dye sample parameters.

For rectangularly-shaped beam profiles with radius $r_{\mathrm{L}}$ the solid angle $\Delta \Omega$ is given by

$$
\Delta \Omega=\pi r_{\mathbf{L}}^{2} / l_{\mathrm{eff}}^{2}
$$

where $l_{\text {eff }}$ is the shorter of either the sample length $l$ or the penetration depth at which the pump pulse intensity is reduced to its $1 / \mathrm{e}$ value. At low pump intensities, $l_{\text {eff }}$ is determined by the ground-state absorption $\left(\sigma_{12} N l_{\text {eff }}=1 ; N\right.$, number density of dye molecules). In an intermediate intensity range 


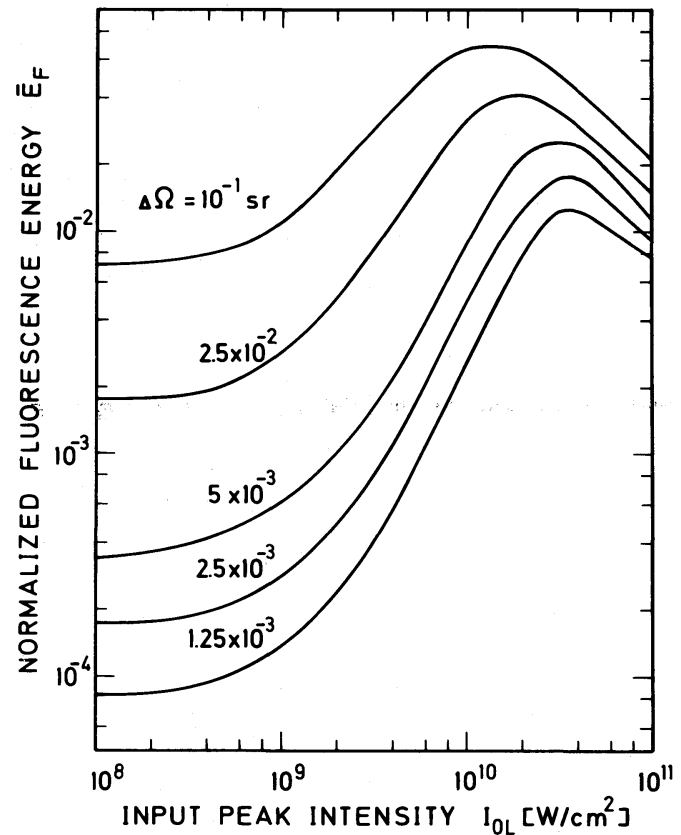

Figure 6 Dependence of energy amplification on solid angle of amplified spontaneous emission.

the bleaching determines $l_{\text {eff }}\left(N l_{\text {eff }}=I_{0 \mathrm{~L}} \Delta t_{\mathrm{L}} / h \nu_{\mathrm{L}}\right)$. At high pump intensities the penetration depth is limited by the excited-state absorption $\left(\sigma_{\text {ex }} N l_{\text {eff }}=1\right)$.

For Gaussian-shaped beam profiles the solid angle is contracted with increased amplification of fluorescence light. In the following the reduction of $\Delta \Omega$ with amplification is estimated. The fluorescence intensity at $l_{\text {eff }}$ is given by

$$
I_{\mathrm{F}}\left(l_{\mathrm{eff}}, r\right)=\text { const. } \times\left\{\exp \left[\left(\sigma_{\mathrm{em}}-\sigma_{\mathrm{ex}}\right) l_{\mathrm{eff}} N_{3}(r)\right]-1\right\} \text { (see Equation } 19 \text { in Section 3.4). }
$$

The population of the fluorescing level is proportional to the pump laser intensity

$$
N_{3}(r) \simeq \text { const. } \times I(r)=\text { const }^{\prime} . \times \exp \left[-\ln 2\left(r / r_{1 / 2}\right)^{2}\right] .
$$

The radius $\tilde{r}$, where the fluorescence intensity reduces to half of its peak value at $r=0$, is determined by

$$
I_{\mathrm{F}}\left(l_{\text {eff }}, \tilde{r}\right) / I_{\mathrm{F}}\left(l_{\text {eff }}, 0\right)=\exp \left\{\kappa \exp \left[-\ln 2\left(\tilde{r} / r_{1 / 2}\right)^{2}\right]-1\right\} /[\exp (\kappa)-1]=1 / 2
$$

where

$$
\kappa=\left(\sigma_{\mathrm{em}}-\sigma_{\mathrm{ex}}\right) N_{3}(r=0) l_{\mathrm{eff}} .
$$

Solving this equation for $\tilde{r}$ leads to [18]

$$
\tilde{r}=r_{1 / 2}(\ln \kappa-\ln \ln \{[\exp (\kappa)+1] / 2\})^{1 / 2} /(\ln 2)^{1 / 2} .
$$

Replacing $r_{\mathrm{L}}$ of Equation 12a by $\tilde{r}$ we find for the solid angle

$$
\Delta \Omega=\frac{\pi r_{1 / 2}^{2}}{l_{\text {eff }}^{2}} \frac{\ln \kappa-\ln \ln \{[\exp (\kappa)+1] / 2\}}{\ln 2} .
$$

It should be noted that $\exp (\kappa)$ is approximately equal to the intensity amplification of the fluorescence signal

$$
\exp (\kappa) \simeq\left[\bar{E}_{\mathrm{F}}\left(I_{\mathrm{L}}\right) / \Delta t_{\mathrm{F}}\left(I_{\mathrm{F}}\right)\right] /\left[\bar{E}_{\mathrm{F}}(0) / \Delta t_{\mathrm{F}}(0)\right] .
$$

At weak fluorescence amplification $(\kappa \rightarrow 0)$ the solid angle reduces to $\Delta \Omega=\pi r_{1 / 2}^{2} / l_{\text {eff }}^{2}$ while at high intensities Equation $12 \mathrm{~b}$ simplifies to $\Delta \Omega=\kappa^{-1}\left(\pi r_{1 / 2}^{2} / l_{\text {eff }}^{2}\right)$. 


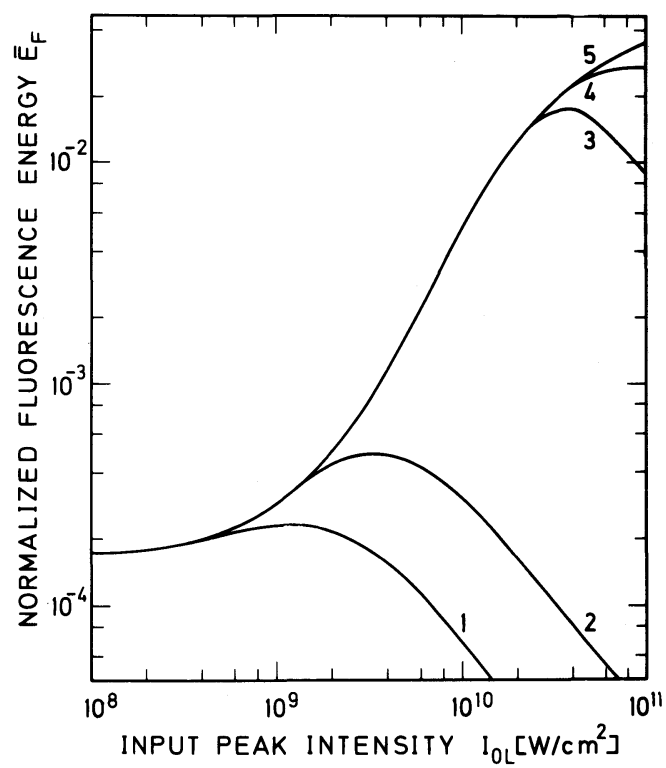

Figure 7 Effect of dye concentration on amplification of fluorescence energy. The concentrations are $2 \times 10^{-5} \mathrm{M}(1), 4 \times 10^{-5} \mathrm{M}(2), 10^{-4} \mathrm{M}(3), 2 \times 10^{-4} \mathrm{M}(4)$, and $4 \times 10^{-4} \mathrm{M}(5)$.

\subsubsection{Effects of dye parameters}

3.2.2.1. The dependence of $\bar{E}_{\mathrm{F}}, \Delta t_{\mathrm{F}}$ and $T_{\mathrm{E}}$ on the dye concentration is plotted in Figs. 7, 8 and 9 , respectively. (A constant $\Delta \Omega=2.5 \times 10^{-3} \mathrm{sr}$ is assumed for all the concentrations). The normalized fluorescence energy $\bar{E}_{\mathrm{F}}$ and the duration $\Delta t_{\mathrm{F}}$ are found to be independent of the dye concentration as long as the pump pulse is not intense enough to excite all the available dye molecules $\left[n_{\text {photon }}(\right.$ laser $\left.)<N\right]$. The maximum $\bar{E}_{\mathrm{F}}$ value and the corresponding pump intensity $I_{0 \mathrm{~L}, \mathrm{opt}}$ are determined by the number density of dye molecules $\left[n_{\text {photon }}(\right.$ laser $\left.) \simeq N\right]$. For $I_{0 \mathrm{~L}}>I_{0 \mathrm{~L}, \text { opt }}$ the normalized fluorescence signal

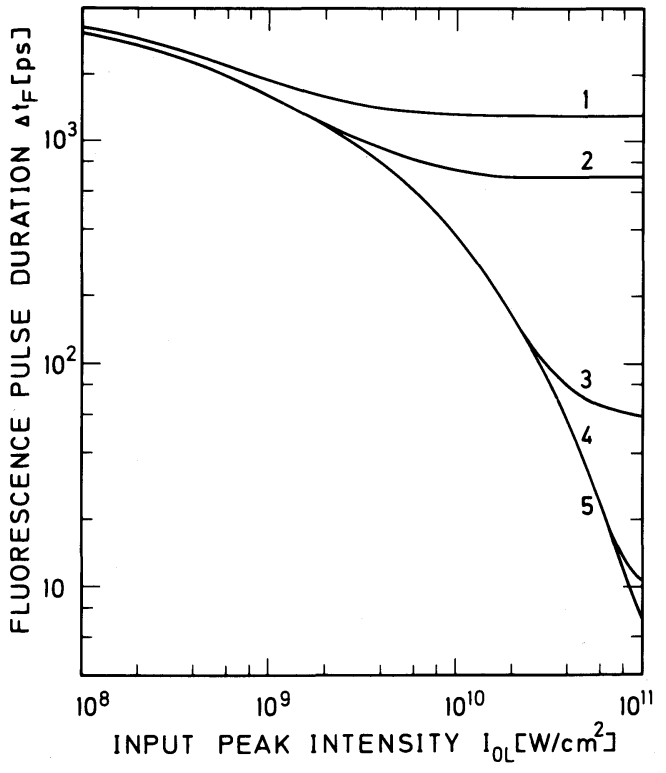

Figure 8 Effect of dye concentration on shortening of fluorescence pulses. The concentrations used are $2 \times 10^{-5} \mathrm{M}(1), 4 \times 10^{-5} \mathrm{M}(2), 10^{-4} \mathrm{M}(3), 2 \times 10^{-4} \mathrm{M}$ (4) and $4 \times 10^{-4} \mathrm{M}(5)$.

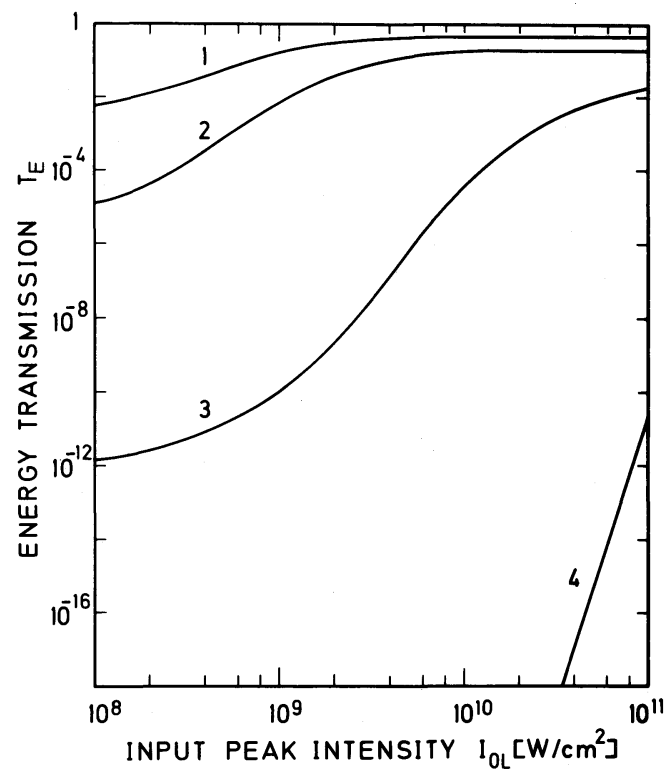

Figure 9 Effect of dye concentration on energy trans-

mission of pump pulse. $1, C=2 \times 10^{-5} \mathrm{M} ; 2, C=$ $4 \times 10^{-5} \mathrm{M} ; 3, C=10^{-4} \mathrm{M} ; 4, C=2 \times 10^{-4} \mathrm{M}$. 


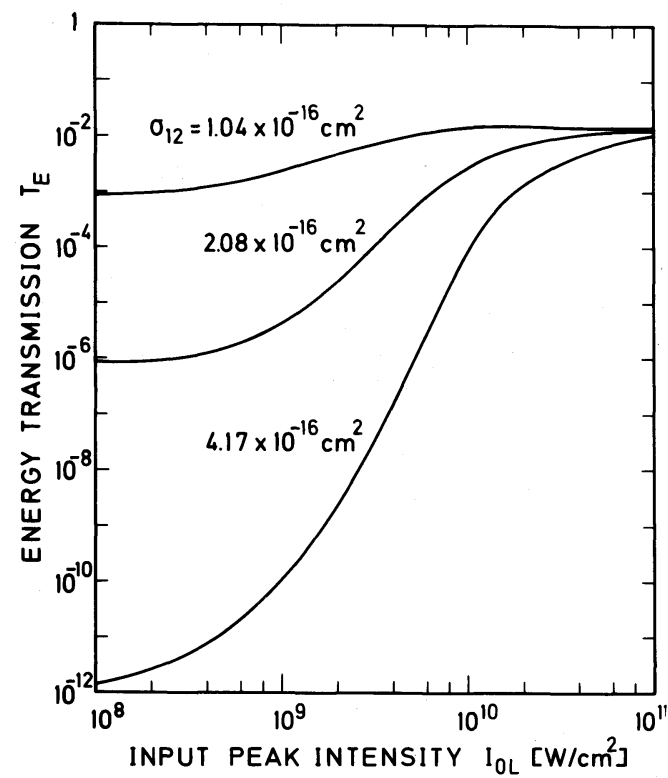

Figure 10 Influence of ground-state absorption on energy transmission of pump pulse.

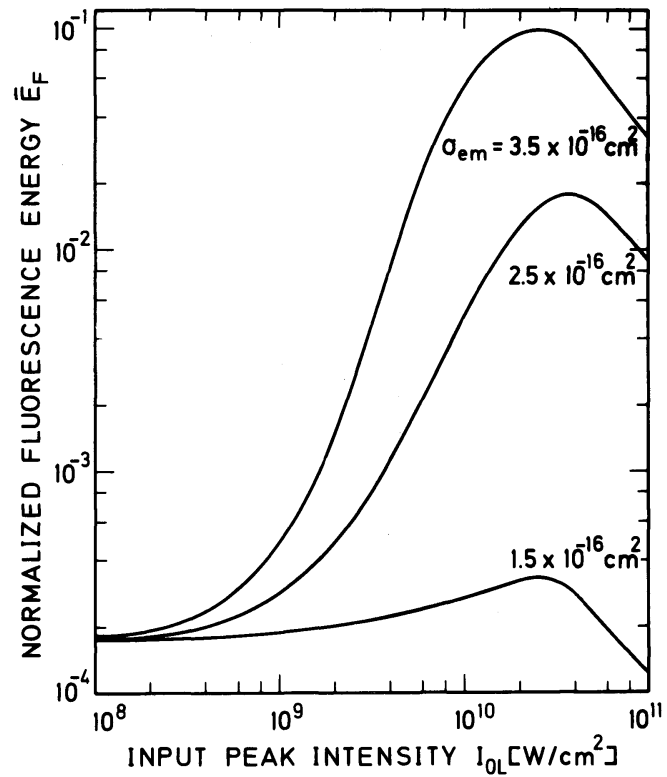

Figure 11 Dependence of normalized fluorescence energy on stimulated-emission cross-section.

$E_{\mathrm{F}}=E_{\mathrm{F}} / E_{\mathrm{abs}}$ decreases due to excited-state absorption of the pump pulse (see later) and $\Delta t_{\mathrm{F}}$ does not shorten any further. High dye concentrations allow a strong amplification of spontaneous emission and a large pulse shortening approaching the pump pulse duration. The energy transmission, $T_{\mathrm{E}}$, reduces drastically with increasing dye concentration since the ground-state absorption and the excited-state absorption are proportional to the population of the respective levels.

3.2.2.2. The effects of the ground-state absorption cross-section, $\sigma_{12}$, on $\bar{E}_{\mathrm{F}}$ and $\Delta t_{\mathrm{F}}$ are negligibly small in the investigated absorption $\left(1 \times 10^{-16} \mathrm{~cm}^{2} \leqslant \sigma_{12} \leqslant 4.2 \times 10^{-16} \mathrm{~cm}^{2}\right)$ and intensity range $\left(10^{8} \mathrm{~W} \mathrm{~cm}^{-2} \leqslant I_{0 \mathrm{~L}} \leqslant 8 \times 10^{10} \mathrm{~W} \mathrm{~cm}^{-2}\right)$. Within the studied region, the whole pump pulse is absorbed in the sample and contributes to the amplification of spontaneous emission. The energy transmission $T_{\mathrm{E}}$ depends exponentially on $\sigma_{12}$ at low pump intensities and is limited by excited-state absorption at high pump intensities (see Fig. 10).

3.2.2.3. The influence of the stimulated emission cross-section $\sigma_{\mathrm{em}}$ on $\bar{E}_{\mathrm{F}}$ and $\Delta t_{\mathrm{F}}$ is studied in Figs. 11 and 12. The energy transmission $T_{\mathrm{E}}$ of the pump pulse is independent of $\sigma_{\mathrm{em}}$. The normalized fluorescence energy $\bar{E}_{\mathrm{F}}$ increases strongly and the fluorescence duration $\Delta t_{\mathrm{F}}$ shortens drastically with increasing $\sigma_{\mathrm{em}}$ values. The maximum value of $\bar{E}_{\mathrm{F}}$ and the limiting $\Delta t_{\mathrm{F}}$ value are determined by the available dye molecules (concentration). The decrease of $\bar{E}_{\mathrm{F}}$ at very high input intensities is caused by excited-state absorption (see Section 3.2.2.4). At high stimulated-emission cross-section the fluorescence duration approaches the pump pulse duration at high pump intensities.

3.2.2.4. The effects of excited-state absorption $\sigma_{\mathrm{ex}}$ on $\bar{E}_{\mathrm{F}}, \Delta t_{\mathrm{F}}$ and $T_{\mathrm{E}}$ are depicted in Figs. 13,14 and 15 , respectively.

Without excited-state absorption $\left(\sigma_{\mathrm{ex}}=0\right)$ the highest amplification, the largest energy transmission and the shortest fluorescence durations are obtained. At high pump intensities all dye molecules are excited and contribute to the emission. A further increase of the pump intensity increases the energy transmission and does not affect the fluorescence behaviour since no additional molecules are present 

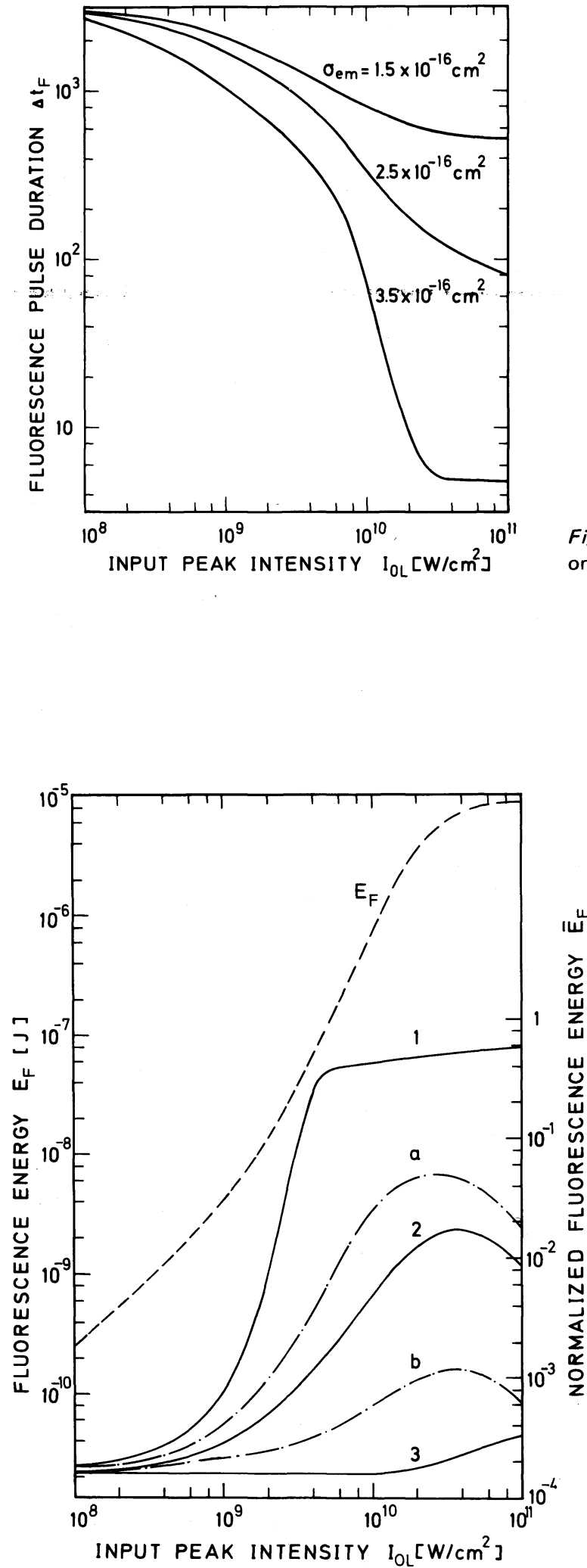

Figure 12 Dependence of fluorescence pulse shortening on stimulatedemission cross-section.

Figure 13 Amplified fluorescence output versus input peak intensity of pump pulse for various excited-state absorptions. Dashed curve shows absolute fluorescence output within $\Delta \Omega=$ $2.5 \times 10^{-3} \mathrm{sr}$ for $\sigma_{\mathrm{ex}, \mathrm{L}}=\sigma_{\mathrm{ex}, \mathrm{F}}=6 \times 10^{-17} \mathrm{~cm}^{2}$ (beam diameter $d_{L}=0.5 \mathrm{~mm}$ ). The solid and dash-dot curves present normalized fluorescence energies. $1, \sigma_{\mathrm{ex}, \mathrm{L}}=\sigma_{\mathrm{ex}, \mathrm{F}}=0 ; 2, \sigma_{\mathrm{ex}, \mathrm{L}}=$ $\sigma_{\mathrm{ex}, \mathrm{F}}=6 \times 10^{-17} \mathrm{~cm}^{2} ; 3, \sigma_{\mathrm{ex}, \mathrm{L}}=\sigma_{\mathrm{ex}, \mathrm{F}}=$ $1.2 \times 10^{-16} \mathrm{~cm}^{2} ; a, \sigma_{\mathrm{ex}, \mathrm{L}}=6 \times 10^{-17} \mathrm{~cm}^{2}$ and $\sigma_{\text {ex }, \mathrm{F}}=0 ; \mathrm{b}, \sigma_{\mathrm{ex}, \mathrm{L}}=6 \times 10^{-17} \mathrm{~cm}^{2}$ and $\sigma_{\mathrm{ex}, \mathrm{F}}=$ $1.2 \times 10^{-16} \mathrm{~cm}^{2}$. 


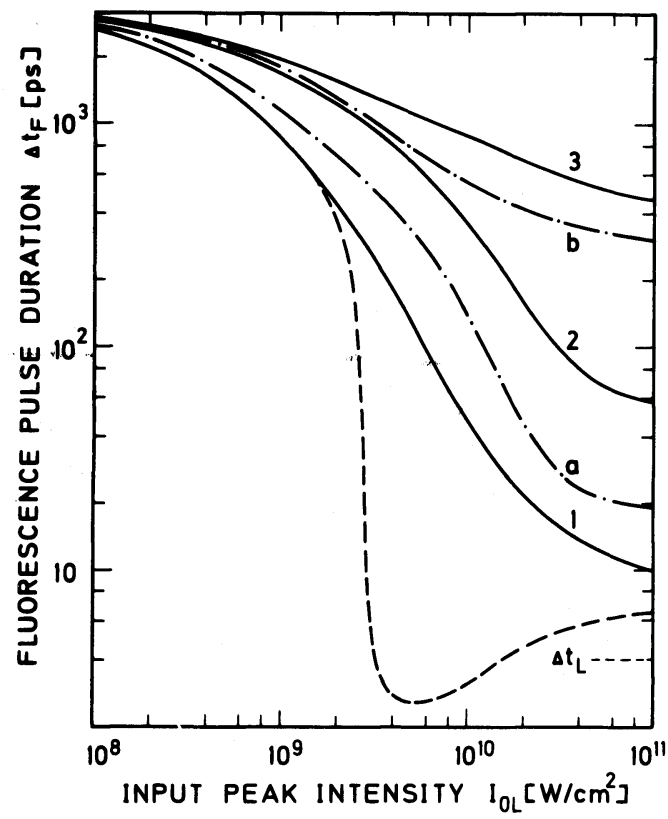

Figure 14 Shortening of fluorescence pulses for various excited-state absorption cross-sections. Dashed curve, $C=2 \times 10^{-4} \mathrm{M}$ and $\sigma_{\mathrm{ex}}=0$ : Solid curves, $C=10^{-4} \mathrm{M}$ and $\sigma_{\mathrm{ex}, \mathrm{L}}=\sigma_{\mathrm{ex}, \mathrm{F}}=\sigma_{\mathrm{ex}}$ with $1, \sigma_{\mathrm{ex}}=0 ; 2$, $\sigma_{\text {ex }}=6 \times 10^{-17} \mathrm{~cm}^{2} ; 3, \sigma_{\text {ex }}=1.2 \times 10^{-16} \mathrm{~cm}^{2}$. Dash-dot curves, $C=10^{-4} \mathrm{M}$ and $\sigma_{\mathrm{ex}, \mathrm{L}} \neq \sigma_{\mathrm{ex}}, \mathrm{F}$ with $\mathrm{a}$, $\sigma_{\text {ex, }}=6 \times 10^{-17} \mathrm{~cm}^{2}$ and $\sigma_{\mathrm{ex}, \mathrm{F}}=0 ; \mathrm{b}, \sigma_{\mathrm{ex}, \mathrm{L}}=$ $6 \times 10^{-17} \mathrm{~cm}^{2}$ and $\sigma_{\mathrm{ex}, \mathrm{F}}=1.2 \times 10^{-16} \mathrm{~cm}^{2}$.

for stimulated emission. At higher dye concentrations the amplification [proportional to $\exp \left(\sigma_{\mathrm{em}} N_{3} l\right)$ ] becomes so large that emission pulses shorter than the pump pulse duration are obtainable (see dashed curve in Fig. 14 for $C=2 \times 10^{-4} \mathrm{M}$ and $\left.\sigma_{\mathrm{ex}}=0\right)$. The minimum fluorescence duration $\left(\Delta t_{\mathrm{F}}<\Delta t_{\mathrm{L}}\right)$ is reached when the pump energy is just large enough to excite all dye molecules towards the end of the excitation pulse $\left(I_{0 \mathrm{~L}} \Delta t_{\mathrm{L}} / h \nu_{\mathrm{L}} \simeq N l\right)$. At higher input peak intensities the dye is already completely excited at the beginning of the excitation pulse and the complete excitation remains for the duration of the pump pulse. As a result, the fluorescence duration lengthens to $\Delta t_{\mathrm{F}}>\Delta t_{\mathrm{L}}$ (see dashed curve in Fig. 14 for $I_{0 \mathrm{~L}}>10^{10} \mathrm{~W} \mathrm{~cm}^{-2}$.

Group dispersion effects may be neglected even when the fluorescence duration approaches the pump pulse duration because of the small spectral shift $\left(\lambda_{L}-\lambda_{F}<60 \mathrm{~nm}\right)$ and short sample length

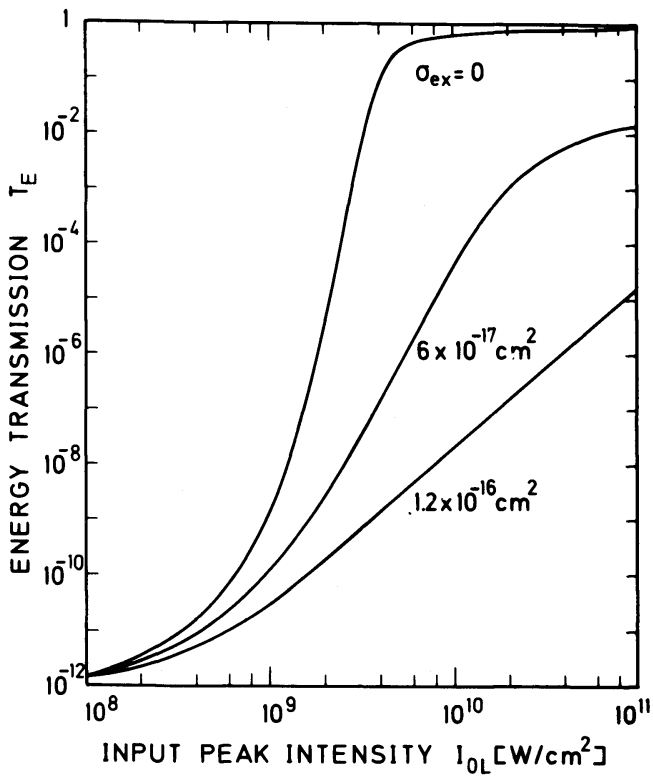

Figure 15 Effect of excited-state absorption on energy transmission of pump pulse. 
$(l=1.13 \mathrm{~cm})$. (The transit time of the fluorescence pulses is only less than $1 \mathrm{ps}$ faster than the pump pulse transit time).

The excited-state absorption of the pump pulse and the fluorescence pulse reduces the amplification and the pulse shortening. The normalized fluorescence energy $\bar{E}_{\mathrm{F}}=E_{\mathrm{F}} / E_{\text {abs }}$ reaches a maximum value at a certain input pulse intensity and decreases at higher pump intensities. The absolute fluorescence energy density $E_{\mathrm{F}}$ remains constant with increasing intensity (see dashed curve in Fig. 13), but the absorbed energy $E_{\text {abs }}$ increases further due to the excitation of higher levels. More than one pump photon is necessary to generate one fluorescence photon because of the excited-state absorption. When the excited-state absorption becomes comparable to the stimulated-emission cross-section, the amplification and pulse shortening diminish. Similarly the energy transmission of the pump pulse decreases with increasing excited-state absorption.

In the discussion given so far, a constant excited-state absorption cross-section is assumed for all molecules in an excited singlet state $\left(\mathrm{S}_{\boldsymbol{n}}, n \geqslant 1\right)$. If a different absorption cross-section $\sigma_{\text {hex }} \neq \sigma_{\mathrm{ex}}$ from Band 6 to Band 7 is chosen (see Fig. 1), Equations 8,10 and 11 have to be modified slightly and an additional equation (Equation 14) for the population density $N_{7}$ is necessary. Equation 4 and the modified equations then become

$$
\begin{aligned}
\frac{\partial N_{6}}{\partial t}= & \sigma_{\text {ex }}\left[\frac{I_{\mathrm{L}}}{h \nu_{\mathrm{L}}}\left(N_{2}+N_{3}-N_{6} \frac{I_{\mathrm{L}}}{I_{\mathrm{L}}+I_{\mathrm{F}}}\right)+\frac{I_{\mathrm{F}}}{h \nu_{\mathrm{F}}}\left(N_{2}+N_{3}-N_{6} \frac{I_{\mathrm{F}}}{I_{\mathrm{L}}-I_{\mathrm{F}}}\right)\right] \\
& -\sigma_{\mathrm{hex}}\left[\frac{I_{\mathrm{L}}}{h \nu_{\mathrm{L}}}\left(N_{6}-N_{7} \frac{I_{\mathrm{L}}}{I_{\mathrm{L}}+I_{\mathrm{F}}}\right)+\frac{I_{\mathrm{F}}}{h \nu_{\mathrm{F}}}\left(N_{6}-N_{7} \frac{I_{\mathrm{F}}}{I_{\mathrm{L}}+I_{\mathrm{F}}}\right)\right] \\
& +k_{76} N_{7}-\left(k_{61}+k_{63}+k_{68}\right) N_{6}(\text { from Equation 8) } \\
\frac{\partial N_{7}}{\partial t}= & \sigma_{\mathrm{hex}}\left[\frac{I_{\mathrm{L}}}{h \nu_{\mathrm{L}}}\left(N_{6}-N_{7} \frac{I_{\mathrm{L}}}{I_{\mathrm{L}}+I_{\mathrm{F}}}\right)+\frac{I_{\mathrm{F}}}{h \nu_{\mathrm{F}}}\left(N_{6}-N_{7} \frac{I_{\mathrm{F}}}{I_{\mathrm{L}}+I_{\mathrm{F}}}\right)\right]-k_{76} N_{7} \\
\frac{\partial I_{\mathrm{L}}}{\partial x}+\frac{\eta}{c_{0}} \frac{\partial I_{\mathrm{L}}}{\partial t}= & -I_{\mathrm{L}}\left(\int _ { 0 } ^ { \pi / 2 } \left\{\left(\sigma_{\| 12} \cos ^{2} \theta+\sigma_{\perp 12} \sin ^{2} \theta\right)\left[N_{1}(\theta)-N_{2}(\theta)\right]+\left(\sigma_{\| \operatorname{lex}} \cos ^{2} \theta\right.\right.\right. \\
& \left.\left.\left.+\sigma_{\perp \text { ex }} \sin ^{2} \theta\right)\left[N_{2}(\theta)+N_{3}-N_{6}\right]\right\} \sin \theta \mathrm{d} \theta+\sigma_{\mathrm{hex}} N_{6}+\sigma_{\mathrm{T}, \mathrm{P}} N_{8}\right) \\
& (\text { from Equation 10) } \\
\frac{\partial I_{\mathrm{F}}}{\partial x}+\frac{\eta}{c_{0}} \frac{\partial I_{\mathrm{F}}}{\partial t}= & \frac{h \nu_{\mathrm{F}} k_{34} N_{3} \Delta \Omega}{4 \pi}+I_{\mathrm{F}}\left[\sigma_{\mathrm{em}}\left(N_{3}-N_{4}\right)-\sigma_{15}\left(N_{1}-N_{5}\right)-\sigma_{\mathrm{ex}}\left(N_{2}+N_{3}-N_{6}\right)\right. \\
& \left.-\sigma_{\text {hex }} N_{6}-\sigma_{\mathrm{T}, \mathrm{P}} N_{8}\right](\text { from Equation 11) }
\end{aligned}
$$

Without absorption from Level 6 to higher-lying states $\left(\sigma_{\text {hex }}=0\right)$ the amplification increases by a factor of 1.6 and the fluorescence pulse duration is shorter by 10 per cent at $I_{0 \mathrm{~L}}=10^{10} \mathrm{~W} \mathrm{~cm}^{-2}$. An increased absorption cross-section $\sigma_{\text {hex }}>\sigma_{\text {ex }}\left(\sigma_{\text {hex }}=4 \times 10^{-16} \mathrm{~cm}^{2}\right)$ reduces the amplification by a factor of 2 and increases the fluorescence duration by 25 per cent at $I_{0 \mathrm{~L}}=10^{10} \mathrm{~W} \mathrm{~cm}^{-2}$. The energy transmission $T_{\mathrm{E}}$ is more drastically influenced by $\sigma_{\text {hex }}\left[T_{\mathrm{E}}\left(\sigma_{\text {hex }}=0\right)=2 \times 10^{-4}\right.$ and $T_{\mathrm{E}}\left(\sigma_{\text {hex }}=4 \times 10^{-16} \mathrm{~cm}^{2}\right)=$ $5 \times 10^{-6}$ at $I_{0 \mathrm{~L}}=10^{10} \mathrm{~W} \mathrm{~cm}^{-2}$ ].

We return to the situation of a constant excited-state absorption cross-section for all molecules in an excited singlet state $\left(\mathrm{S}_{n}, n \geqslant 1\right)$; but now we study the influence of different excited-state absorption cross-sections $\sigma_{\mathrm{ex}, \mathrm{L}}$ and $\sigma_{\mathrm{ex}, \mathrm{F}}$ for the pump laser photons and the emission photons. (In the Equations 5,6 and 8 the terms with $\sigma_{\mathrm{ex}} I_{\mathrm{L}}$ and $\sigma_{\mathrm{ex}} I_{\mathrm{F}}$ change to $\sigma_{\mathrm{ex}, \mathrm{L}} I_{\mathrm{L}}$ and $\sigma_{\mathrm{ex}, \mathrm{F}} I_{\mathrm{F}}$, respectively). The dash-dot curves in Figs. 13 and 14 are calculated with a fixed value of $\sigma_{\text {ex, L }}=6 \times 10^{-17} \mathrm{~cm}^{2}$ while $\sigma_{\text {ex, F }}$ is assumed to be 0 (Curves a) and $1.2 \times 10^{-16} \mathrm{~cm}^{2}$ (Curves b). The excited-state absorption of the fluorescence light reduces the amplification and the pulse shortening. The energy transmission $T_{\mathrm{E}}$ of the pump pulse is not influenced by absorption of the fluorescence pulse. 


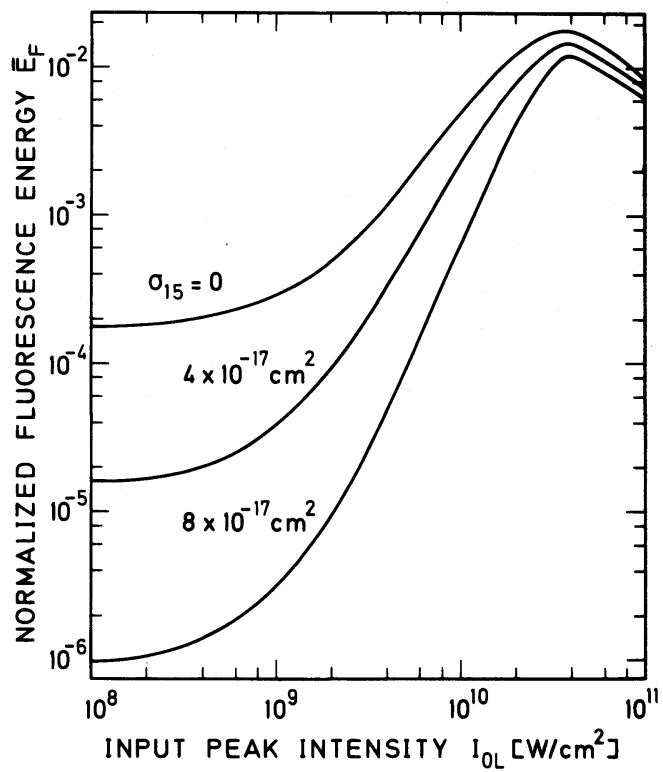

Figure 16 Influence of reabsorption on normalized fluorescence energy.

3.2.2.5. The effect of reabsorption of fluorescence light on the normalized fluorescence energy $\bar{E}_{\mathrm{F}}$ is depicted in Fig. 16. At low pump intensities the ground-state population $N_{1}$ is large and the fluorescence light is strongly reabsorbed for $\sigma_{15} \neq 0$. With increasing pump intensity the population $N_{1}$ is reduced and correspondingly the reabsorption effect. The fluorescence signal approaches the value obtained in the case without reabsorption. The duration of the emission is only weakly influenced by the reabsorption (see solid curves in Fig. 5b). The shortening effect is slightly stronger when reabsorption is present. It is known from conventional fluorescence spectroscopy that reabsorption lengthens the fluorescence decay time [32], i.e. the reabsorbed fluorescence light is reemitted at a later time. In our calculations this effect is not observed since we only study the reabsorption along the sample length within the solid angle $\Delta \Omega$ and assume no reabsorption outside this angle. The energy transmission $T_{\mathrm{E}}$ is not affected by the reabsorption of fluorescence light. In the experiments $\sigma_{15}$ depends on the pump pulse intensity since the fluorescence spectrum shifts to shorter wavelengths with increasing laser intensity (see Section 3.2.2.8 below).

3.2.2.6. The influence of the spontaneous emission rate $k_{34}\left(=k_{3}\right)$ on $\bar{E}_{\mathrm{F}}, \Delta t_{\mathrm{F}}$ and $T_{\mathrm{E}}$ is studied in Fig. 17. The curves are presented for $I_{0 \mathrm{~L}}=10^{10} \mathrm{~W} \mathrm{~cm}^{-2}$. For $k_{34}<10^{9} \mathrm{~s}^{-1}, \bar{E}_{\mathrm{F}}, \Delta t_{\mathrm{F}} / \tau_{\mathrm{F}}$ and $T_{\mathrm{E}}$ are found to be independent of $k_{34}\left(\tau_{F}=1 / k_{3}\right.$ is the spontaneous fluorescence decay time). At faster spontaneous decay rates the population in Level 3 is reduced quickly and the stimulated emission becomes weaker (smaller $\bar{E}_{\mathrm{F}}$ and larger $\Delta t_{\mathrm{F}} / \tau_{\mathrm{F}}$ values). The fast refilling of the ground state reduces the energy transmission of the pump pulse. At short fluorescence decay times $\tau_{F}=1 / k_{3}=1 / k_{34}$ the amplified spontaneous emission signal may become shorter than the pump pulse.

When the nonradiative decay rate $k_{31}$ becomes comparable to the radiative rate $k_{34}$, the number of emitting molecules $N_{3}$, the amplification and the pulse shortening are reduced.

The inter-system crossing from Level 3 to the triplet state 8 (rate $k_{38}$ ) has a similar reducing effect as the internal conversion rate $k_{31}$. In Fig. 18 the dependence of $\bar{E}_{\mathrm{F}}$ and $\Delta t_{\mathrm{F}}$ on $k_{38}$ is presented for $I_{0 \mathrm{~L}}=10^{10} \mathrm{~W} \mathrm{~cm}^{-2}$. The amplification and the pulse shortening [see curve representing $\Delta t_{\mathrm{F}} / \tau_{\mathrm{F}}=$ $\left.\Delta t_{\mathrm{F}}\left(k_{31}+k_{34}+k_{38}\right)\right]$ are reduced because molecules are lost for emission. The effect of the intersystem crossing rate on the amplified spontaneous emission is rather weak (less than linear) since the transfer time is long compared to the duration of the amplified spontaneous signal. The energy transmission is independent of $k_{38}$ within the studied range. 

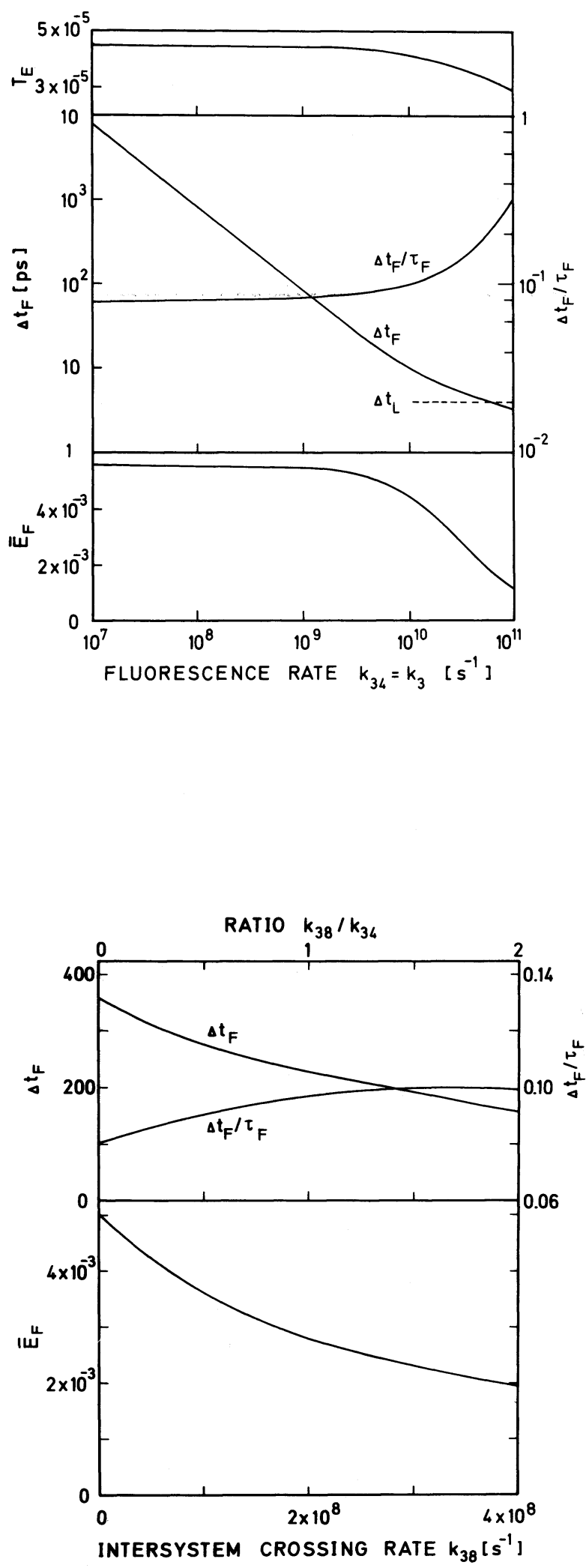

Figure 17 Effect of spontaneous emission rate $k_{34}$ on amplified spontaneous emission and energy transmission of pump pulse. $k_{31}=k_{38}=0 \cdot \tau_{\mathrm{F}}=$ $1 / k_{3}$ is the fluorescence decay time. $l_{0 \mathrm{~L}}=$ $10^{10} \mathrm{~W} \mathrm{~cm}^{-2}$.
Figure 18 Influence of inter-system crossing rate $k_{38}$ on amplified spontaneous emission. $\tau_{\mathbf{F}}=$ $\left(k_{31}+k_{34}+k_{38}\right)^{-1} ; k_{31}=2.5 \times 10^{7} \mathrm{~s}^{-1} ; k_{34}=$ $2 \times 10^{8} \mathrm{~s}^{-1} \cdot I_{0 \mathrm{~L}}=10^{10} \mathrm{~W} \mathrm{~cm}^{-2}$. 


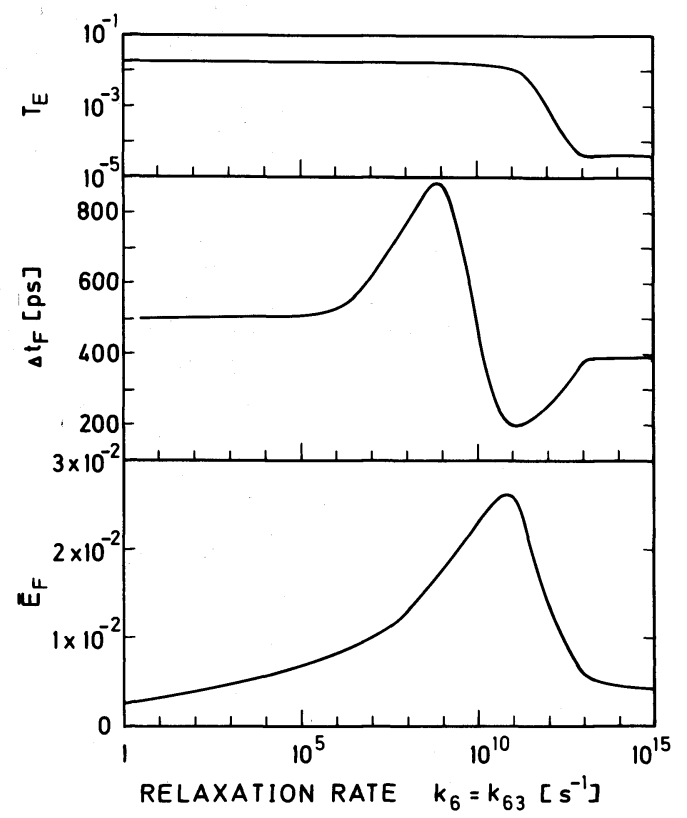

Figure 19 Influence of the relaxation $k_{6}$ from higher excited singlet states on amplified spontaneous emission and energy transmission. $k_{61}=k_{68}=0 . l_{0 \mathrm{~L}}=$ $10^{10} \mathrm{Wcm}^{-2}$.

3.2.2.7. The decay of higher excited molecules to the emitting Level 3 , to the ground state (1), and to the triplet or decomposition states (8) strongly influence the amplification of spontaneous emission and the resulting pulse shortening. Up to now we assumed that all higher excited molecules relax to the fluorescing Level 3 with a decay rate of $k_{63}=10^{13} \mathrm{~s}^{-1}$. In the following, the influence of the various decay channels and decay rates is studied.

(a) The effects of the decay rate $k_{63}=k_{6}$ on $\bar{E}_{\mathrm{F}}, \Delta t_{\mathrm{F}}$ and $T_{\mathrm{E}}$ are studied in Fig. 19 for $I_{0 \mathrm{~L}}=$ $10^{10} \mathrm{~W} \mathrm{~cm}^{-2}$. Starting at low transition rates the amplification increases with rising $k_{63}$ since the relaxation to Level 3 increases the amount of fluorescing molecules. As long as $k_{63}$ is shorter than $1 / \Delta t_{\mathrm{L}}$ the energy transmission is not affected by the relaxation. When $k_{63}$ becomes comparable to $1 / \Delta t_{\mathrm{L}}$ the energy transmission $T_{\mathrm{E}}$ is reduced by the repeated absorption of laser light. As a consequence $\bar{E}_{\mathrm{F}}=$ $E_{\mathrm{F}} / E_{\text {abs }}$ goes down. For $k_{63} \gg 1 / \Delta t_{\mathrm{L}}$ the energy transmission $T_{\mathrm{E}}$ and the normalized fluorescence energy $\bar{E}_{\mathrm{F}}$ tend to a constant value.

The dependence of the fluorescence duration $\Delta t_{F}$ on the relaxation rate $k_{63}$ is shown in the middle part of Fig. 19. For $k_{63}<1 / \Delta t_{\mathrm{F}}$ the fluorescence duration $\Delta t_{\mathrm{F}}$ remains approximately constant. When $k_{63}$ becomes comparable to $1 / \Delta t_{\mathrm{F}}$, the fluorescence duration is increased by the relaxing molecules from Level 6. A further increase of $k_{63}$ results in a shortening of $\Delta t_{\mathrm{F}}$ because of the increased amplification of fluorescence light. Finally at rates $k_{63}>1 / \Delta t_{\mathrm{L}}$ the laser absorption increases, the fluorescence amplification reduces and the pulse duration $\Delta t_{\mathrm{F}}$ is increased to a stationary value.

The strong dependence of $\bar{E}_{\mathrm{F}}, \Delta t_{\mathrm{F}}$ and $T_{\mathrm{E}}$ on $k_{63}=k_{6}$ may be used to determine $k_{63}$ when all other parameters of the dye are known. For $k_{6} \gg \Delta t_{\mathrm{L}}^{-1}$ or $k_{6}<\Delta t_{\mathrm{L}}^{-1}$ the energy transmission becomes independent of $k_{6}$ and a determination of $\sigma_{\mathrm{ex}, \mathrm{L}}$ without exact knowledge of $k_{6}$ is possible. The change of $T_{\mathrm{E}}$ with $k_{6}$ in the region $\Delta t_{\mathrm{L}}^{-1}<k_{6}<40 \Delta t_{\mathrm{L}}^{-1}$ may be used to determine $k_{6}$ and $\sigma_{\text {ex, }}$ by varying $\Delta t_{\mathrm{L}}$.

(b) The amplification of fluorescence light and the fluorescence pulse shortening are reduced when the molecules return directly from Level 6 to the ground state. The molecules do not return to the emitting level effecting the amplified spontaneous emission. In Fig. 20 the intensity dependence of $\bar{E}_{\mathrm{F}}$ for $k_{61}=k_{6}=10^{13} \mathrm{~s}^{-1}$ (Curve 3) is compared with the case of $k_{63}=k_{6}=10^{13} \mathrm{~s}^{-1}$ (Curve 1). The same comparison is made in Fig. $21 \mathrm{~b}$ for the fluorescence duration $\Delta t_{\mathrm{F}}$. The molecules returned to the ground state are ready for a new pump pulse absorption and reduce its energy transmission (Curve a in Fig. 23). The involvement of the relaxed molecules in the excitation process increases the population of the 


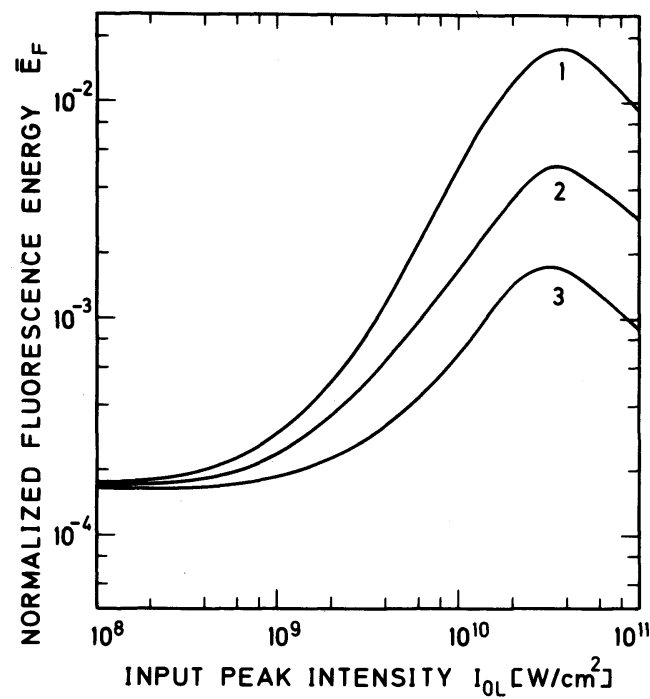

Figure 20 Dependence of normalized fluorescence energy on relaxations of higher excited states $(6)$ within the singlet system. Curve $1: k_{6}=k_{63}=10^{13} \mathrm{~s}^{-1}, k_{61}=$ $k_{68}=0$. Curve 2: $k_{6}=10^{13} \mathrm{~s}^{-1}, k_{63}=5 \times 10^{12} \mathrm{~s}^{-1}$, $k_{61}=5 \times 10^{12} \mathrm{~s}^{-1}, k_{68}=0$. Curve $3: k_{6}=k_{61}=10^{13} \mathrm{~s}^{-1}$, $k_{63}=k_{68}=0$.

fluorescing level and is responsible for the larger amplification and pulse shortening as compared to the case where the molecules are transferred to a triplet state or are decomposed.

The intermediate situation where some of the molecules relax from Level 6 to Level 3 and some to Level $1\left(k_{63}=k_{61}=5 \times 10^{12} \mathrm{~s}^{-1}\right)$ is presented in the Curves 2 of Fig. 20 and Fig. $21 \mathrm{~b}$. In this case the energy transmission lies between Curve a and Curve 1 of Fig. 23.

(c) In Figs. 21a, 22 and 23 the effect of the transfer of higher excited molecules (Level 6) to triplet levels and decomposed states (Level 8) is clarified. Molecules that leave the singlet level system are lost for fluorescence emission. Consequently the amplification and pulse shortening are reduced. At higher pump intensities the excited-state absorption becomes very effective and the transfer to triplet states or decomposed states increases correspondingly. The fluorescence signal reduces in this case, even below the low intensity value (Fig. 22), and the fluorescence duration is enlarged to the small signal value (Fig. 21a). If no absorption of laser light is assumed for the triplet molecules and decomposed mole-

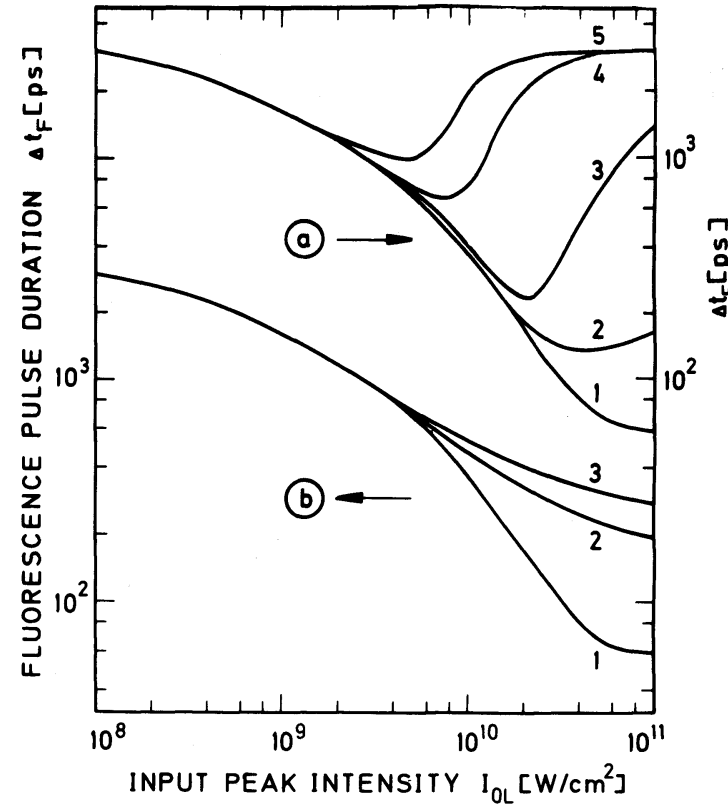

Figure 21 Dependence of fluorescence pulse shortening on relaxation of higher excited singlet states $(6)$. $k_{6}=k_{61}+k_{63}+k_{68}=10^{13} \mathrm{~s}^{-1}$ for all curves. (a) Decay to triplet system or to photodecomposed states. $k_{61}=0.1, k_{68}=0 ; 2, k_{68}=10^{11} \mathrm{~s}^{-1} ; 3, k_{68}=10^{12} \mathrm{~s}^{-1}$; $4, k_{68}=5 \times 10^{12} \mathrm{~s}^{-1} ; 5, k_{68}=10^{13} \mathrm{~s}^{-1}$. (b) Relaxation within the singlet system. $k_{68}=0.1, k_{63}=10^{13} \mathrm{~s}^{-1} ; 2$, $k_{61}=k_{63}=5 \times 10^{12} \mathrm{~s}^{-1} ; 3, k_{61}=10^{13} \mathrm{~s}^{-1}$. 


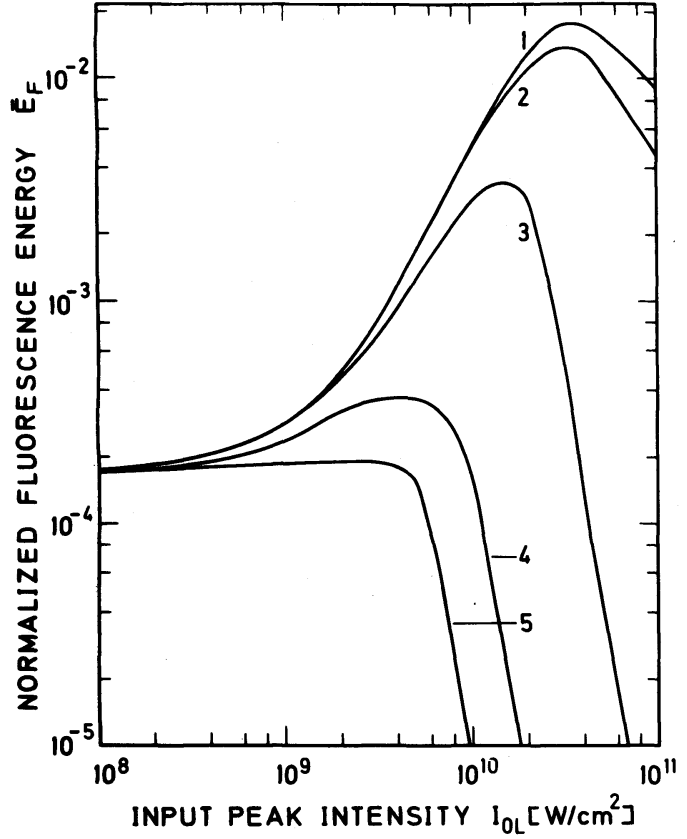

Figure 22 Dependence of normalized fluorescence energy on relaxation of higher excited states to triplet or decomposed states. $1, k_{68}=0 ; 2, k_{68}=10^{11} \mathrm{~s}^{-1} ; 3, k_{68}=$ $10^{12} \mathrm{~s}^{-1} ; 4, k_{68}=5 \times 10^{12} \mathrm{~s}^{-1} ; 5, k_{68}=10^{13} \mathrm{~s}^{-1}$. For all curves is $k_{6}=10^{13} \mathrm{~s}^{-1}$ and $k_{61}=0$.

cules (as depicted in the figures) the energy transmission grows up to high values (Fig. 23). The assumption of absorption by the transferred molecules $\left(\sigma_{\mathrm{T}, \mathrm{P}} \neq 0\right)$ reduces the amplification of spontaneous emission and the pulse shortening even further.

The drastic dependence of $\bar{E}_{\mathrm{F}}, \Delta t_{\mathrm{F}}$ and $T_{\mathrm{E}}$ on $k_{68}$ allows an accurate determination of this parameter by comparing the calculation with experimental results.

3.2.2.8. In the calculations described the influences of a spectral distribution of the ground-state absorption cross-section and of the emission cross-section are not included. We discuss these effects only briefly. The frequency dependence of $\sigma_{\mathrm{em}}=\sigma_{\mathrm{em}}(v)$ results in a spectral narrowing of the amplified

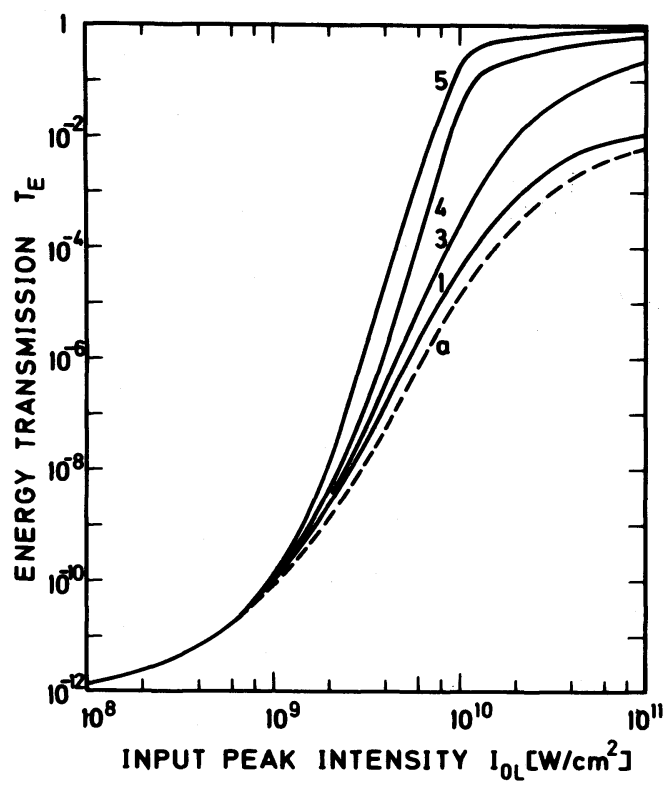

Figure 23 Effect of relaxation rates from higher excited states (6) on the energy transmission of the pump pulse. $1, k_{63}=10^{13} \mathrm{~s}^{-1} ; 3, k_{63}=9 \times 10^{12} \mathrm{~s}^{-1}, k_{68}=10^{12} \mathrm{~s}^{-1}$; $4, k_{63}=k_{68}=5 \times 10^{12} \mathrm{~s}^{-1} ; 5, k_{68}=10^{13} \mathrm{~s}^{-1} ; \mathrm{a}, k_{61}=$ $10^{13} \mathrm{~s}^{-1}$. For all curves $k_{6}=10^{13} \mathrm{~s}^{-1}$. 
spontaneous emission, because the frequency components with the largest emission cross-section are most effectively amplified. The narrowing of the spectral width $\Delta \nu_{\mathrm{F}}$ is approximately given by [18-20] (see also derivation of Equation 12b)

$$
\Delta \nu_{\mathrm{F}}\left(I_{\mathrm{L}}\right)=\Delta \nu_{\mathrm{F}}(0) \sqrt{\left(\frac{\ln \kappa^{\prime}-\ln \ln \left\{\left[\exp \left(\kappa^{\prime}\right)+1\right] / 2\right\}}{\ln 2}\right)}
$$

where $\kappa^{\prime}=\left(\sigma_{\mathrm{em}, \max }-\sigma_{\mathrm{ex}}\right) N_{3}\left(I_{\mathrm{L}}\right) l$. At high input laser intensities $\left[N_{3}\left(I_{\mathrm{L}}\right) \rightarrow N, \kappa \gg 1\right]$ Equation $17 \mathrm{a}$ reduces to

$$
\Delta \nu_{\mathbf{F}}\left(I_{\mathbf{L}}\right)=\kappa^{-1 / 2} \Delta \nu_{\mathbf{F}}(0)
$$

For a $10^{-4} \mathrm{M}$ dye solution of $1 \mathrm{~cm}$ length with $\sigma_{\mathrm{em}, \max }-\sigma_{\mathrm{ex}}=2 \times 10^{-16} \mathrm{~cm}^{2}$ the spectral narrowing is limited to $\Delta \nu_{\mathrm{F}}\left(I_{\mathrm{L}}\right) / \Delta \nu_{\mathrm{F}}(0) \geqslant 0.3$.

The frequency distribution of the ground-state absorption cross-section influences the fluorescence emission. The absorption edge at the long frequency side shifts the emission spectrum to longer wavelength. The shift increases with dye concentration. The frequency of maximum fluorescence emission $\nu_{\mathrm{F}, \max }$ appears at a longer wavelength than expected from the frequency $\nu_{\mathrm{em}, \max }$ of maximal stimulated-emission cross-section. As intense pump pulses bleach the ground state, the reabsorption is reduced and the frequency $\nu_{\mathrm{F}, \max }$ of maximum fluorescence emission shifts towards the frequency $\nu_{\mathrm{em}, \max }$ of optimum $\sigma_{\mathrm{em}}$.

The spectral narrowing and the spectral shift of the fluorescence emission are observed experimentally as described in [17].

\subsection{Determination of dye parameters by comparison with experiment}

The theoretical results are compared with the experimental measurements on rhodamine $6 \mathrm{G}$ and rhodamine B in [17]. The values of $k_{6}, k_{68} / k_{6}, k_{63} / k_{6}, \sigma_{\mathrm{ex}, \mathrm{L}}$ and $\sigma_{\mathrm{ex}, \mathrm{F}}$ that enter the calculations of amplified spontaneous emission are determined in [17]. The measurement of $T_{E}$ for two different pulse durations $\Delta t_{\mathrm{L}}$ allows the determination of $k_{6}$ and $\sigma_{\mathrm{ex}, \mathrm{L}}$ (see Fig. 19). $k_{6} \gtrsim 10^{13} \mathrm{~s}^{-1}$ (see also. [31]) and $\sigma_{\text {ex, } L} \simeq(5 \pm 1) \times 10^{-17} \mathrm{~cm}^{2}$ were found for both rhodamine $6 \mathrm{G}$ and rhodamine $\mathrm{B}$. The strong dependence of $\bar{E}_{\mathrm{F}}$ and $\Delta t_{\mathrm{F}}$ on $k_{68} / k_{6}$ (see Fig. 21a and 22) is used in its determination. Values of $k_{68} / k_{6}=$ $0.01 \pm 0.01$ and $0.02 \pm 0.01$ were found for rhodamine $6 \mathrm{G}$ and rhodamine $\mathrm{B}$, respectively. The unknown ratio $k_{63} / k_{6}$ is determined by comparing the $\mathrm{S}_{1}-\mathrm{S}_{0}$ fluorescence yields after excitation with the second harmonic and the fourth harmonic of a mode-locked Nd-glass laser. In the case of rhodamine $6 \mathrm{G}$, a value of $k_{63} / k_{6}=0.95 \pm 0.05$ was found while the result for rhodamine B was $k_{63} / k_{6}=0.7 \pm 0.05$. The $\mathrm{S}_{1}-\mathrm{S}_{n}$ excited-state absorption cross-section $\sigma_{\mathrm{ex}, \mathrm{F}}$ of the fluorescence light was obtained by fitting the calculations to the measured $\bar{E}_{\mathrm{F}}$ values. The measured values were $\sigma_{\mathrm{ex}, \mathrm{F}} \simeq(9 \pm 3) \times 10^{-17} \mathrm{~cm}^{2}$ for rhodamine $6 \mathrm{G}$ and rhodamine $\mathrm{B}$.

\subsection{Analytical estimates of dye behaviour}

The described studies of amplified spontaneous emission set the limits for experiments where the amplification of spontaneous emission may be neglected. In addition they give the necessary conditions under which intense picosecond light pulses at new frequencies may be generated by amplified spontaneous emission.

\subsubsection{Conditions for negligible amplified spontaneous emission}

We present some simplified analytical expressions for the conditions where amplification of spontaneous emission (superfluorescence) is negligibly small and conventional analysis of fluorescence spectra leads to correct results. When the populations of Levels 4 and 6 and the reabsorption of fluorescence light are neglected Equation 11 simplifies to

$$
\partial I_{\mathrm{F}} / \partial z=I_{\mathrm{F}}\left(\sigma_{\mathrm{em}}-\sigma_{\mathrm{ex}}\right) N_{3}+h \nu_{\mathrm{F}} k_{34} N_{3} \Delta \Omega / 4 \pi
$$


The solution for $N_{3}=$ constant is

$$
I_{\mathrm{F}}(z)=\frac{h \nu_{\mathrm{F}} k_{34} \Delta \Omega}{4 \pi\left(\sigma_{\mathrm{em}}-\sigma_{\mathrm{ex}}\right)}\left\{\exp \left[\left(\sigma_{\mathrm{em}}-\sigma_{\mathrm{ex}}\right) N_{3} z\right]-1\right\}
$$

For $\sigma_{\mathrm{ex}}>\sigma_{\mathrm{em}}$ Equation 14 changes to

$$
I_{\mathrm{F}}(z)=\frac{h \nu_{\mathrm{F}} k_{34} \Delta \Omega}{4 \pi\left(\sigma_{\mathrm{ex}}-\sigma_{\mathrm{em}}\right)}\left\{1-\exp \left[-\left(\sigma_{\mathrm{ex}}-\sigma_{\mathrm{em}}\right) N_{3} z\right]\right\} .
$$

Equation 20 shows that the fluorescence signal decays to zero with increasing sample length when $\sigma_{\mathrm{ex}}>\sigma_{\mathrm{em}}$. In this case no amplification occurs.

When $\sigma_{\mathrm{em}}=\sigma_{\mathrm{ex}}$ Equation 19 reduces to

$$
I_{\mathrm{F}}(z)=\left(h \nu_{\mathrm{F}} k_{34} \Delta \Omega / 4 \pi\right) N_{3} z .
$$

This result is identical to spontaneous emission as is seen by solving Equation 18 without the first term. Amplification of spontaneous emission does not occur.

When $\sigma_{\mathrm{em}}>\sigma_{\mathrm{ex}}$ amplification of spontaneous emission takes places. The maximum amplification factor at high pump intensities is easily estimated by assuming complete bleaching of the dye. The condition for $N_{3}$ is $N_{3} \leqslant N$ where $N$ is the total number density of dye molecules. The amplification of spontaneous emission is estimated to be

$$
\frac{I_{\mathrm{F}}(z)}{I_{\text {spont }}(z)} \leqslant \frac{\exp \left[\left(\sigma_{\mathrm{em}}-\sigma_{\mathrm{ex}}\right) N z\right]-1}{N z\left(\sigma_{\mathrm{em}}-\sigma_{\mathrm{ex}}\right)} .
$$

The last equation is very useful for determining the dye concentration and sample length that may be used without being affected by amplification of spontaneous emission. As an example the amplification is less than 2 at any pump intensity for $\sigma_{\mathrm{em}}-\sigma_{\mathrm{ex}}=2 \times 10^{-16} \mathrm{~cm}^{2}, z=1 \mathrm{~cm}$ and $C=10^{-5} \mathrm{M}^{-1}(N=$ $6 \times 10^{15} \mathrm{~cm}^{-3}$ ).

\subsubsection{Conditions for optimum amplification of spontaneous emission}

The most effective amplification of spontaneous emission is obtained for dyes with negligible excitedstate absorption and high stimulated-emission cross-section. Fluorescence pulse durations as short as the pump pulse duration, and even shorter, are possible with high dye concentrations and intense pump pulses. Dyes with short fluorescence decay times approach the pump pulse duration at lower pump intensities or lower dye concentration.

The pulse shortening may be estimated by studying the depopulation of the emitting level. The fluorescence light decays slightly faster as is seen in Fig. 2 and 3 and by analysing Equation 19. $I_{\mathrm{F}}(t)$ depends exponentially on $N_{3}(t)$; the mathematical situation is identical to the spectral narrowing, where $I_{\mathrm{F}}(\nu)$ depends exponentially on $\sigma_{\mathrm{em}}(\nu)$, or to the narrowing of divergence which is studied in Section 3.2.1.2 where $I_{\mathrm{F}}(r)$ is proportional to $\exp \left[\left(\sigma_{\mathrm{em}}-\sigma_{\mathrm{ex}}\right) l_{\mathrm{eff}} N_{3}(r)\right]$.

Neglecting the population of Levels 4 and 6, Equation 5 simplifies for $t>\Delta t_{\mathrm{L}}$ to

$$
\frac{\partial N_{3}}{\partial t}=-\left[k_{3}+\frac{\sigma_{\mathrm{em}} I_{\mathrm{F}}}{h \nu_{\mathrm{F}}}\right] N_{3}
$$

where $k_{3}=k_{31}+k_{34}+k_{38}=1 / \tau_{\mathrm{F}}$. The solution is

$$
N_{3}(t)=N_{30} \exp \left\{-\left[k_{3}+\frac{\sigma_{\mathrm{em}} I_{\mathrm{F}}}{h \nu_{\mathrm{F}}}\right] t\right\} .
$$

The emitting level depopulates with a time constant

$$
\tau_{3}=1 /\left[k_{3}+\sigma_{\mathrm{em}} I_{\mathrm{F}}(z) / h \nu_{\mathrm{F}}\right] .
$$

For $\sigma_{\mathrm{em}} I_{\mathrm{F}} / h \nu_{\mathrm{F}}<k_{3}$ the stimulated emission of fluorescence light is too weak to shorten the 
spontaneous decay rate $k_{3}=k_{31}+k_{34}+k_{38}$. By introduction of Equation 19 into Equation 25, the effects of the dye parameters and pump parameters on the depopulation of Level 3 are readily seen. With $\tau_{\mathrm{F}}=1 / k_{3}$ and $q_{\mathrm{F}}=k_{34} / k_{3}$ we find

$$
\tau_{3}=\frac{\tau_{\mathrm{F}}}{1+q_{\mathrm{F}} \frac{\Delta \Omega}{4 \pi}\left\{\exp \left[\left(\sigma_{\mathrm{em}}-\sigma_{\mathrm{ex}}\right) N_{3} z\right]-1\right\}\left[\sigma_{\mathrm{em}} /\left(\sigma_{\mathrm{em}}-\sigma_{\mathrm{ex}}\right)\right]} .
$$

The shortest $\tau_{3}$ values are obtained at high pump intensities where $N_{3}$ may approach the total population density $N$. Equation 26 indicates that a minimum dye concentration is necessary to obtain appreciable pulse shortening and to approach the pump pulse duration (see also Fig. 8).

As a numerical example we estimate the minimum dye concentration that is necessary to generate fluorescence pulses with a duration approximately equal to the pump pulse duration. Using the values $z=1 \mathrm{~cm}, \tau_{\mathrm{F}} \simeq 4 \mathrm{~ns}, q_{\mathrm{F}} \simeq 1, \sigma_{\mathrm{em}}-\sigma_{\mathrm{ex}} \simeq 2 \times 10^{-16} \mathrm{~cm}^{2}, \Delta \Omega \simeq 10^{-3} \mathrm{sr}$ and $\tau_{3}=\Delta t_{\mathrm{L}}=4 \mathrm{ps}$ we calculated a minimum dye concentration $N \geqslant N_{3}=8 \times 10^{16} \mathrm{~cm}^{-3} \simeq 1.4 \times 10^{-4} \mathrm{M}$. For these numbers we calculate with the aid of Equation 22 a fluorescence amplification $I_{\mathrm{F}}(z) \simeq 10^{6} I_{\mathrm{spont}}(z)$ and $E_{\mathrm{F}}(z) \simeq 10^{3} E_{\text {spont }}(z)$.

The minimum pump intensity necessary to excite $N_{3} z \simeq N z$ molecules $\mathrm{cm}^{-2}$ is estimated from the balance $N_{3} z \simeq\left(I_{0 \mathrm{~L}} \Delta t_{\mathrm{L}} / h \nu_{\mathrm{L}}\right) \exp \left(-\sigma_{\mathrm{ex}} N_{3} z\right)$. The last term takes into account the fact that more than one laser photon has to be absorbed to generate a fluorescing molecule due to the excited-state absorption. The minimum laser intensity is found to be

$$
I_{0 \mathrm{~L}} \geqslant \frac{N_{3} z h \nu_{\mathrm{L}} \exp \left(\sigma_{\mathrm{ex}} N_{3} z\right)}{\Delta t_{\mathrm{L}}}
$$

The necessary laser intensity depends strongly on the excited-state absorption cross-section $\sigma_{\mathrm{ex}}$. For $\sigma_{\mathrm{ex}}=0, I_{0 \mathrm{~L}} \simeq 8 \times 10^{9} \mathrm{~W} \mathrm{~cm}^{-2}$ is enough to generate fluorescence-pulse durations equal to the pump pulse durations (numerical values of above this were used; compare with dashed curve in Fig. 14). The minimum pump intensity for $\Delta t_{\mathrm{F}} \simeq \tau_{3} \simeq \Delta t_{\mathrm{L}}$ increases to $I_{0 \mathrm{~L}} \simeq 9 \times 10^{11} \mathrm{~W} \mathrm{~cm}^{-2}$ when a value of $\sigma_{\mathrm{ex}}=6 \times 10^{-17} \mathrm{~cm}^{2}$ is assumed for the dye. The excited-state absorption drastically reduces the fluorescence pulse shortening. (At $I_{0 \mathrm{~L}}>10^{11} \mathrm{~W} \mathrm{~cm}^{-2}$ other nonlinear optical effects in the dye solution can no longer be neglected).

In the estimates above we did not take into account the relaxation rate $k_{68}$ that removes molecules from the singlet system. The amplification of fluorescence is drastically reduced when a remarkable percentage of molecules is accumulated in the loss level $N_{8}$. With some approximations the population of Level 8 may be estimated. Equations 8 and 9 may be simplified to

$$
\begin{gathered}
\frac{\partial N_{6}}{\partial t}=\sigma_{\mathrm{ex}}\left(\frac{I_{\mathrm{L}}}{h \nu_{\mathrm{L}}}+\frac{I_{\mathrm{F}}}{h \nu_{\mathrm{F}}}\right)\left(N_{2}+N_{3}\right)-k_{6} N_{6} \\
\frac{\partial N_{8}}{\partial t}=k_{68} N_{6} .
\end{gathered}
$$

We want to determine $N_{6}$ and $N_{8}$ at the end of the pump pulse $t=\Delta t_{\mathrm{L}}$. The pump pulse should be rectangularly shaped within $0<t<\Delta t_{\mathrm{L}} . I_{\mathrm{F}}$ may be neglected. The populations of Levels 2 and 3 are given by $N_{2}+N_{3} \simeq I_{\mathrm{L}} t /\left(h \nu_{\mathrm{L}}\right)$ as long as $I_{\mathrm{L}} t / h \nu_{\mathrm{L}} \leqslant N$. With these assumptions the solution of Equation 28 is (for $\left.k_{6}>\Delta t_{\mathrm{L}}^{-1}\right)$

$$
N_{6}(t) \simeq \begin{cases}\sigma_{\mathrm{ex}}\left(I_{\mathrm{L}} / h \nu_{\mathrm{L}}\right)^{2} t / k_{6} & \text { for } k_{6}^{-1}<t<\Delta t_{\mathrm{L}} \\ \sigma_{\mathrm{ex}}\left(I_{\mathrm{L}} / h \nu_{\mathrm{L}}\right) N / k_{6} & \text { for } I_{\mathrm{L}} t / h \nu_{\mathrm{L}}>N\end{cases}
$$

The solution of Equation 29 is found by use of Equation 30 


$$
N_{8}(t)=k_{68} \int_{0}^{t} N_{6}\left(t^{\prime}\right) \mathrm{d} t^{\prime}= \begin{cases}\left(k_{68} / k_{6}\right) \sigma_{\mathrm{ex}}\left(I_{\mathrm{L}} / h \nu_{\mathrm{L}}\right)^{2} t^{2} / 2 & \text { for } k_{6}^{-1}<t<\Delta t_{\mathrm{L}} \\ \left(k_{68} / k_{6}\right) \sigma_{\mathrm{ex}}\left(I_{\mathrm{L}} / h \nu_{\mathrm{L}}\right) N t & \text { for } I_{\mathrm{L}} t / h \nu_{\mathrm{L}}>N .\end{cases}
$$

The population $N_{8}(t)$ is proportional to the ratio $k_{68} / k_{6}$. Equation 26 may be used to estimate the effects of loss on the amplified spontaneous emission. As an example, we find that $N_{8}$ approaches $N$ at the end of the pump pulse $\left(t=\Delta t_{\mathrm{L}}=4 \mathrm{ps}\right)$ for $\sigma_{\mathrm{ex}}=6 \times 10^{-17} \mathrm{~cm}^{2}, I_{\mathrm{L}}=5 \times 10^{10} \mathrm{~W} \mathrm{~cm}^{-2}$ and $k_{68} / k_{6}=0.03$. (Compare these numbers with Curve 3 in Fig. 22). The drastic reduction of amplified spontaneous emission with $\left(k_{68} / k_{6}\right) ; \sigma_{\mathrm{ex}}$ and $I_{\mathrm{L}} \Delta t_{\mathrm{L}}$ is clearly seen.

\section{Summary}

The amplification of spontaneous emission was studied with a multi-level model. The various effects of pump and dye parameters were thoroughly discussed. The theoretical results agree with experimental data and allow the determination of interesting molecular parameters. The calculations lead to experimental restrictions that have to be met if spectroscopic studies are not be complicated by amplified spontaneous emission. On the other hand, the necessary dye parameters and experimental conditions for the generation of intense picosecond light pulses at new frequencies by amplification of spontaneous emission were clearly elaborated.

\section{Acknowledgements}

The authors are very indebted to Professors W. Kaiser and M. Maier for many valuable discussions.

\section{References}

1. M. E. MACK, Appl. Phys. Lett. 15 (1969) 166-8.

2. C. V. SHANK, A. DIENES and W. T. SILFVAST, ibid 17 (1970) 307-9.

3. M. M. MALLEY and P. M. RENTZEPIS, Chem. Phys. Lett. 7 (1970) 57-60.

4. S. L. CHIN and G. BEDARD, Opt. Comm. 3 (1971) 299-300.

5. Idem, ibid 4 (1971) 148-9.

6. J. SCHUBERT, Phys. Lett. 34A (1971) 381-2.

7. M. R. TOPP, P. M. RENTZEPIS and R. P. JONES, Chem. Phys. Lett. 9 (1971) 1-5.

8. C. LIN, T. K. GUSTAFSON and A. DIENES, Opt. Comm. 8 (1973) 210-5.

9. L. Allen and G. I. PETERS, Phys. Rev. A8 (1973) 2031-47.

10. Y. SigiURA. Y. MATSUNAGA and T. FUJIOKA, J. Appl. Phys. 45 (1974) 4969-70.

11. U. GANIEL, A. HARDY, G. NEUMANN and D. TREVES, IEEE J. Quant. Elect. QE-11 (1975) 881-92.

12. A. N. RUBINOV, M. C. RICHARDSON, K. SALA and A. J. ALCOCK, Appl. Phys. Lett. 27 (1975) 358-60.

13. P. BURLAMACCHI, R. PRATESI and U. VANNI, Appl. Opt. 15 (1976) 2684-9.

14. G. R. FLEMING, A. E. W. KNIGHT, J. M. MORRIS, R. J. ROBBINS and G. W. ROBINSON, Chem. Phys. 23 (1977) 61-70.

15. H. E. LESSING, E. LIPPERT and W. RAPP, Chem. Phys. Lett. 7 (1970) 247-53.

16. H. E. LESSING, Opt. Quant. Elect. 8 (1976) 309-15.

17. W. FALKENSTEIN, A. PENZKOFER and W. KAISER, to be published.

18. A. YARIV, 'Quantum Electronics' (Wiley, New York, 1975).

19. L. W. CASPERSON and A. YARIV, IEEE J. Quant. Elect. QE-8 (1972) 80-5.

20. L. W. CASPERSON, Opt. Comm. 8 (1973) 85-7.

21. P. P. FEOFILOV, 'The physical basis of polarized emission' (Consultants Bureau, New York, 1961).

22. A. PENZKOFER and W. FAL KENSTEIN, Chem. Phys. Lett. 44 (1976) 547-52.

23. L. D. LANDAU and E. M. LIFSCHITZ, 'Klassiche Feld theorie' (Adakemie-Verlag, Berlin, 1966) p. 194.

24. T. J. CHUNG and K. B. EISENTHAL, Chem. Phys. Lett. 11 (1971) 368-70.

25. A. PENZKOFER, W. FAL KENSTEIN and W. KAISER, ibid 44 (1976) 82-7.

26. G. MOUROU, IEEE J. Quant. Elect. QE-11 (1975) 1-8.

27. F. P. SCHÄFER, 'Dye Lasers' (Springer, New York, 1973) p. 35.

28. D. N. DEM PSTER, T. MORROW and M. F. QUINN, J. Photochem. 2 (1973) 343-59.

29. D. RICARD and J. DUCUING, J. Chem. Phys. 62 (1975) 3616-9.

30. A. V. ARISTOV and Yu. S. MASLYY KOV, Opt. Spectr. 41 (1976) 141-3.

31. C. V. SHANK, E. P. IPPEN and O. TESCHKE, Chem. Phys. Lett. 45 (1977) 291-4.

32. J. B. BIR KS, 'Photophysics of aromatic molecules' (Wiley, Interscience, New York, 1970) p. 92. 